\title{
Tissue characterization with depth- resolved attenuation coefficient and backscatter term in intravascular optical coherence tomography images
}

Shengnan Liu

Yohei Sotomi

Jeroen Eggermont

Gaku Nakazawa

Sho Torii

Takeshi Ijichi

Yoshinobu Onuma

Patrick W. Serruys

Boudewijn P. F. Lelieveldt

Jouke Dijkstra 


\title{
Tissue characterization with depth-resolved attenuation coefficient and backscatter term in intravascular optical coherence tomography images
}

\author{
Shengnan Liu, ${ }^{\text {a }}$ Yohei Sotomi, ${ }^{b}$ Jeroen Eggermont, ${ }^{a}$ Gaku Nakazawa, ${ }^{c}$ Sho Torii, $^{\mathrm{c}}$ Takeshi Ijichi, ${ }^{\mathrm{c}}$

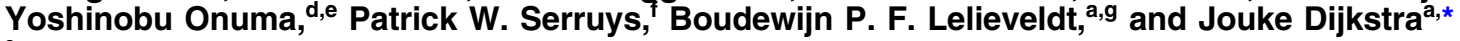 \\ aLeiden University Medical Center, Division of Imaging Processing, Department of Radiology, Leiden, The Netherlands \\ bUniversity of Amsterdam, Academic Medical Center, Amsterdam, The Netherlands \\ 'Tokai University School of Medicine, Department of Cardiology, Kanaagawa, Japan \\ dThoraxcenter, Erasmus Medical Center, Rotterdam, The Netherlands \\ eCardialysis, Rotterdam, The Netherlands \\ IInternational Centre for Circulatory Health, the National Heart and Lung Institute, Imperial College London, London, United Kingdom \\ ${ }^{9}$ Delft University of Technology, Department of Intelligent Systems, Delft, The Netherlands
}

\begin{abstract}
An important application of intravascular optical coherence tomography (IVOCT) for atherosclerotic tissue analysis is using it to estimate attenuation and backscatter coefficients. This work aims at exploring the potential of the attenuation coefficient, a proposed backscatter term, and image intensities in distinguishing different atherosclerotic tissue types with a robust implementation of depth-resolved (DR) approach. Therefore, the DR model is introduced to estimate the attenuation coefficient and further extended to estimate the backscatter-related term in IVOCT images, such that values can be estimated per pixel without predefining any delineation for the estimation. In order to exclude noisy regions with a weak signal, an automated algorithm is implemented to determine the cut-off border in IVOCT images. The attenuation coefficient, backscatter term, and the image intensity are further analyzed in regions of interest, which have been delineated referring to their pathology counterparts. Local statistical values were reported and their distributions were further compared with a two-sample $t$-test to evaluate the potential for distinguishing six types of tissues. Results show that the IVOCT intensity, DR attenuation coefficient, and backscatter term extracted with the reported implementation are complementary to each other on characterizing six tissue types: mixed, calcification, fibrous, lipid-rich, macrophages, and necrotic core. () The Authors. Published by SPIE under a Creative Commons Attribution 3.0 Unported License. Distribution or reproduction of this work in whole or in part requires full attribution of the original publication, including its DOI. [DOI: 10 .1117/1.JBO.22.9.096004]
\end{abstract}

Keywords: depth-resolved; intravascular optical coherence tomography; attenuation coefficient; backscatter term; calcification; lipid; necrotic core; fibrous; macrophages.

Paper 170366PRR received Jun. 6, 2017; accepted for publication Aug. 21, 2017; published online Sep. $12,2017$.

\section{Introduction}

Optical coherence tomography (OCT) is an imaging modality with a high resolution. ${ }^{1}$ Since the light can be transmitted via an optical fiber, OCT has been introduced into intravascular imaging by embedding an optical fiber into an imaging catheter. ${ }^{2}$ The acquired intravascular OCT (IVOCT) images with a high resolution (10 to $20 \mu \mathrm{m}$ ) boosted the understanding of the composition of atherosclerosis in the coronary artery, which is one of the fatal diseases that is responsible for most of the death in the world. ${ }^{3}$ In IVOCT images, different tissue types can be recognized, ${ }^{4}$ e.g., the fibrous tissue is bright, both lipid-rich and calcified plaque appear as low intensities, while the border of the former tends to be diffused and the latter is sharper.

To achieve automated analysis of IVOCT images for diagnosis and risk assessment, much attention has been spent on the

*Address all correspondence to: Jouke Dijkstra, E-mail: J.Dijkstra@lumc.nl quantitative characterization of the tissue composition of the coronary vessel wall. ${ }^{4-15}$ Inspired by the literature on tissue optics in general OCT, ${ }^{16-21}$ one of the important features that has been analyzed is the OCT-derived attenuation and backscatter coefficient. For accurate estimation, the modeling of the transmitting light through biological tissue usually requires taking into consideration the complex interactions ${ }^{18,19}$ such as multiple scatter and concentration dependency. On the other hand, estimations have been reported at an adequate accuracy for tissue quantification based on a simplified model involving a single exponential decaying term. ${ }^{20,21}$

Based on the simplified model, one of the commonly used approaches for the estimation of the attenuation is the least square fitting either an exponential curve to a one-dimensional (1-D) OCT signal or a linear function to the logarithm of 1-D OCT signal. The curve-fitting (CF) attenuation has been widely used to quantify biological tissue such as bladder urothelial carcinoma, ${ }^{22}$ human skin, ${ }^{23}$ nasopharyngeal carcinoma cells, ${ }^{24}$ collagen content in ovarian tissue, ${ }^{25}$ breast tissue, ${ }^{26}$ and human atrial tissue. ${ }^{27}$ Specifically for the analysis of tissue in the 
coronary arteries, an earlier work of Xu et al. ${ }^{8}$ has shown that the $\mathrm{CF}$ approach can be used to estimate both the attenuation and backscatter coefficients, which can improve the differentiation of atherosclerotic tissue. In order to improve the reproducibility and the robustness of the $\mathrm{CF}$ approach, Gargesha et al. ${ }^{28}$ estimated the attenuation coefficient within volumes of interest. The method has been validated with different combinations of noise reduction filtering and outlier rejection to optimize a goodness-of-fit. Furthermore, attenuation and backscatter coefficients were analyzed to characterize the neointima formation after the stent implemented from different manufacturers. ${ }^{29,30}$ van Soest et al. ${ }^{9}$ proposed an automated optimization scheme to estimate the attenuation coefficient by expanding fitting windows within regions assumed to be homogeneous. This approach has been validated with a later study from the same group. ${ }^{31}$ Since the automated algorithm allows for a high robustness and reproducibility, the CF-attenuation is increasingly used for region-based tissue characterization combined with machine learning tools, such as random forest, ${ }^{32}$ Gaussian likelihood map, ${ }^{26}$ and supported vector machine. ${ }^{27}$

Another approach for the estimation of attenuation coefficient has been recently introduced by Vermeer et al. ${ }^{10}$ It is based on a depth-resolved (DR) model and allows for local pixel-wise estimation with two fundamental assumptions, the light should be attenuated completely and the backscatter and attenuation are linearly related to each other. Despite strict assumptions that have been made, their measurements did show a good consistency in both homogeneous and layered phantom experiments. This DR attenuation has been used for the detection of the cerebral edema in OCT images. ${ }^{33}$ Taking the confocal function and the fall-off of the system into consideration, the DR model has been reported to estimate the attenuation more accurately and potentially further improve the pixel-wise tissue characterization in combination with a noise removal approach. ${ }^{34}$ In addition to estimating the attenuation, it should be noted that the DR model has been widely used for the enhancement of OCT images. ${ }^{35-39}$ Due to its recent introduction, no quantitative analysis with the DR model for tissue characterization has been reported on IVOCT images.

The aim of this work is to explore the potential of DR attenuation coefficient, backscatter term, and image intensities in distinguishing different atherosclerotic tissue types with a robust implementation of the DR approach. In order to achieve this goal, the DR attenuation is introduced to IVOCT, the model is further extended to estimate a backscatter term, and a cutoff border is determined to remove noisy regions. Six types of tissue were labeled by two pathologists referring to their histological counterparts. Combining with the image intensities, the estimated attenuation coefficient and the backscatter term are analyzed for the differentiation of these six types of tissues. To the best of our knowledge, no such study has been published so far for intravascular tissue analysis.

The structure of this paper is organized as follows. In Sec. 2.1, the light propagation model and the DR-attenuation are introduced, the estimation of a backscatter term is derived in Sec. 2.2, and the DR model is combined with the CF approach to determine the cut-off depth border in Sec. 2.3. The experimental materials and the implementation of the experiment are described in Sec. 3. The results are reported in Sec. 4 and further discussed in Sec. 5, where the estimated values are extensively compared with that in the literature. The conclusion is drawn in Sec. 6.

\section{Depth-Resolved Model Analysis}

We continue to use the model of light transmission as it has been introduced in the work of Vermeer et al. ${ }^{10}$

$I_{b}(d) \cong \beta \cdot L_{0} \cdot \mu_{b}(d) \cdot e^{-2 \int_{0}^{d} \mu_{t}(u) \mathrm{d} u}$,

where $I_{b}(d)$ is the digitalized signal detected at depth $d, \beta$ accounts for both the quantum efficiency of the detector ${ }^{19}$ and the converting factor of the digitizer, $L_{0}$ is the source light power $\left(\mathrm{W} \mathrm{m}^{-2}\right)$, the attenuation is denoted as $\mu_{t}$, and the backscatter coefficient is denoted as $\mu_{b}$.

\subsection{Depth-Resolved Attenuation}

With an assumption that the backscattered light is a fixed fraction to the attenuated light $\mu_{b}(d)=\alpha \mu_{t}(d)$ the integration from $d$ to infinite of Eq. (1) will be

$$
\begin{aligned}
\int_{d}^{\infty} I_{b}(u) \mathrm{d} u & \cong-\frac{\alpha \beta L_{0}}{2} e^{-2 \int_{0}^{u} \mu(v) \mathrm{d} v}+\left.C\right|_{d} ^{\infty} \\
& =-\frac{I_{b}(u)}{2 \mu_{t}(u)}+\left.C\right|_{d} ^{\infty}=\frac{I_{b}(d)}{2 \mu_{t}(d)}
\end{aligned}
$$

The fraction of Eqs. (1) and (2) is then the estimated attenuation

$\mu_{t}(d) \cong \frac{I_{b}(d)}{2 \int_{d}^{\infty} I_{b}(u) \mathrm{d} u}$.

The attenuation can be estimated with the OCT image intensities as

$\mu[i, j] \approx \frac{I[i, j]}{2 \Delta \sum_{y=j+1}^{\infty} I[i, y]}$,

where an OCT image is denoted as $I$ with size of $M \times N, i$ indicates the A-line number (pixel), $j$ indicates the depth number (pixel), and $\Delta$ is the pixel size (mm/pixel). In order to avoid the accumulation of noise during the calculation, the image is first denoised with a median filter.

\subsection{Backscatter Term Estimation}

Suppose an A-line from a homogeneous region starts at depth $d_{1}$ and ends at depth $d_{2}$. Within this region, both the attenuation and the backscatter coefficients at each depth $d \in\left(d_{1}, d_{2}\right)$ are the same $\mu_{t}(d)=\tilde{\mu}_{t}$ and $\mu_{b}(d)=\tilde{\mu}_{b}$, thus Eq. (1) can be simplified as

$I_{b}(d) \cong L^{\prime} \cdot \tilde{\mu}_{b} \cdot e^{-2 \cdot r \cdot \tilde{\mu}_{t}}$,

where $r=d-d_{1}$ and $r \in\left[0, d_{2}-d_{1}\right]$. The term $L^{\prime} \beta=$ $L_{0} \cdot e^{-2 \int_{0}^{d_{1}} \mu_{t}(u) \mathrm{d} u}$ is the diminished source intensity along the path from the catheter to the defined region. Using the same notation, $L^{\prime}$ is the diminished light power that enters at depth $d_{1}$ with the same unit as $L_{0}\left(\mathrm{~W} \mathrm{~m}^{-2}\right)$ divided by the imaging factor $\beta$.

Within the homogeneous region, $\tilde{\mu}_{t}$ and $\tilde{\mu}_{b}$ can be estimated as the slope and the offset of the linear function fitted with $\ln \left[I_{b}(d) / \beta L^{\prime}\right]$, as it has been proposed that in the work of Schmitt et al. ${ }^{16}$ Noting that the $L^{\prime}$ is a constant relative to $d$, linear fitting $\ln \left[I_{b}(d)\right]$ gives the same estimation of the 
attenuation coefficient. However, the estimation of $L^{\prime}$ is necessary for the estimation of backscatter coefficient.

With the DR model, we consider $d_{1}$ and $d_{2}$ are the start and the end of a pixel, then $e^{-2 \cdot r \cdot \tilde{\mu}_{t}}$ approximates to 1 since $r$ is then very small. Dividing Eq. (5) by a factor of $e^{-2 \int_{0}^{d_{1}} \mu_{t}(u) \mathrm{d} u}$ and taking the logarithm, we get a similar intersection to be our backscatter term as

$T_{b}(d)=\ln \frac{L_{0}}{\beta} \cdot \tilde{\mu}_{b} \cong \ln I_{b}(d)+2 \int_{0}^{d_{1}} \tilde{\mu}_{t}(u) \mathrm{d} u$,

where $\tilde{\mu}_{t}(u)$ is the estimated attenuation at depth $u$. The defined term $T_{b}(d)$ is linear related to the logarithm of the backscatter coefficient with a constant offset related to the initial incident energy $L_{0}$ and the imaging parameter $\beta$.

\subsection{DR-CF Cut-Off Algorithm}

One of the main concerns regarding the pixel-wise DR algorithm is the assumption that the integral of intensity should be taken to infinity. However, infinity in practice is usually substituted with a large value, the maximum depth $D$ of the signal in the case of OCT images. This causes little effect on the estimated attenuation of the superficial tissue region, where the signal is much stronger than the noise. However, as it approaches the end of an A-line, the estimated attenuation coefficient with the noise-dominant signal will be exaggerated and thus is unreliable.

In order to avoid this, we applied the DR-CF algorithm to determine a cut-off depth. Noting that

$$
\int_{d}^{\infty} I_{b}(u) \mathrm{d} u=\frac{\alpha \beta L_{0}}{2} e^{-2 \int_{0}^{d_{1}} \mu(u) \mathrm{d} u} e^{-2 \int_{d_{1}}^{d} \tilde{\mu} \mathrm{d} u}=\frac{\alpha L^{\prime}}{2} e^{-2 r \tilde{\mu}},
$$

thus taking the natural logarithm to the both sides, Eq. (7) becomes

$$
\ln \left[\int_{d}^{\infty} I_{b}(u) \mathrm{d} u\right] \cong-2 r \tilde{\mu}+\ln \frac{\alpha L^{\prime}}{2} .
$$

The second term is a constant, the logarithm of the integral intensities follows a linear function with respect to the depth within this region, and the attenuation can be calculated from the slope. With the DR-CF algorithm, the left term is always decreasing, thus it guarantees that the estimated values are positive and the high noise tolerance of the $\mathrm{CF}$-approach is maintained. Since the deeper signals are influenced strongly by noise, it is difficult to perceive the local variations, but the general decreasing trend can still be estimated with the DR-CF algorithm.

The cut-off algorithm is described in Algorithm 1. The linear fitting is implemented in MATLAB (R2016a, The MathWorks, Inc., Natick, Massachusetts) with the least square approximation. In Algorithm 1 step 2, the size of the sliding window size is chosen to be 21 pixels for each A-line. After fitting, an error image $I_{\text {err }}$ is calculated, and then a threshold of the squared error $5 \times 10^{5}$ was experimentally chosen to create a binary image $I_{\mathrm{bw}}$ in step 3 . In order to determine a continuous border with the regional maximum got in step 3, a morphological closing of $I_{\mathrm{bw}}$ is used in step 4, where the octagon operator is used with a radius of 6 pixels.
Algorithm 1 Determine the DR-CF cut off border.

1. Calculate the $\operatorname{cum} I_{b}=\int_{d}^{D} I_{b}(u) \mathrm{d} u$;

2. From the backside of each A-line, estimate the attenuation with linear fitting according to Eq. (8) with a sliding window calculate the image of the fitting error $I_{\mathrm{err}}$ (the total norm of fitting residuals) is found.

3. Threshold the fitting error and extract a binary image $I_{\mathrm{bw}}$ marking the regional maximum.

4. Morphologically close $I_{\mathrm{bw}}$ with a octagon of radius $R$, and the backborder defined binary image is the defined cut-off depth border.

\section{Experiment and Implementation}

In order to analyze the potential of the IVOCT-estimated optical values for distinguishing the coronary artery tissue, six types of tissues were delineated according to their pathological counterparts harvested from two cadaver hearts, resulting in 135 of IVOCT images for the analysis. The data and the implementation for the analysis are described in more detail as follows.

\subsection{Data Description}

Coronary segments were dissected from the cadaver hearts and imaged ex vivo by optical frequency domain imaging (OFDI). The samples were pinned on a rubber sheet and immersed in a water bath with $0.9 \%$ saline solution. A 0.014 -in. guidewire was introduced into the vessel followed by an OFDI catheter (LUNAWAVE, Terumo). Sequential images were acquired at a pullback rate of $20 \mathrm{~mm} / \mathrm{s}$ (158 frames/s, 512 A-lines per frame). The source light has a center wavelength of $1300 \mathrm{~nm}$ and a bandwidth of $124 \mathrm{~nm}$. The depth resolution for each A-line used in this work is $5.4 \mu \mathrm{m}$ per pixel, which has been calculated with the refractive index stored in the raw data as 1.447 .

\subsubsection{Tissue preparation and plaque assessment}

After the intracoronary imaging, samples were x-rayed and then decalcified using ethylene diamine tetraacetic acid as required. Coronary segments were sectioned at 3- to 4-mm intervals and embedded in paraffin to obtain 4- to $6-\mu$ m-thick sections followed by hematoxylin and eosin (H\&E) and Movat pentachrome stain.

\subsubsection{Matching of IVOCT and histology, and differentiation and delineation of specific tissue type on histology and IVOCT}

In each pathological cross section, two pathologists $(\mathrm{CN}$ and ST) identified the following tissue categories in histology: lipid, calcification, mixed calcified plaque, necrotic core, and macrophage. The remaining tissue was identified as fibrous plaque. The identified tissues in histology were independently delineated by the pathologists such that each region of interest (ROIs) included only one type of tissue [Figs. 1(a)-1(d)].

The histological cross section and the corresponding IVOCT cross section were matched using the lumen shape and tissue 


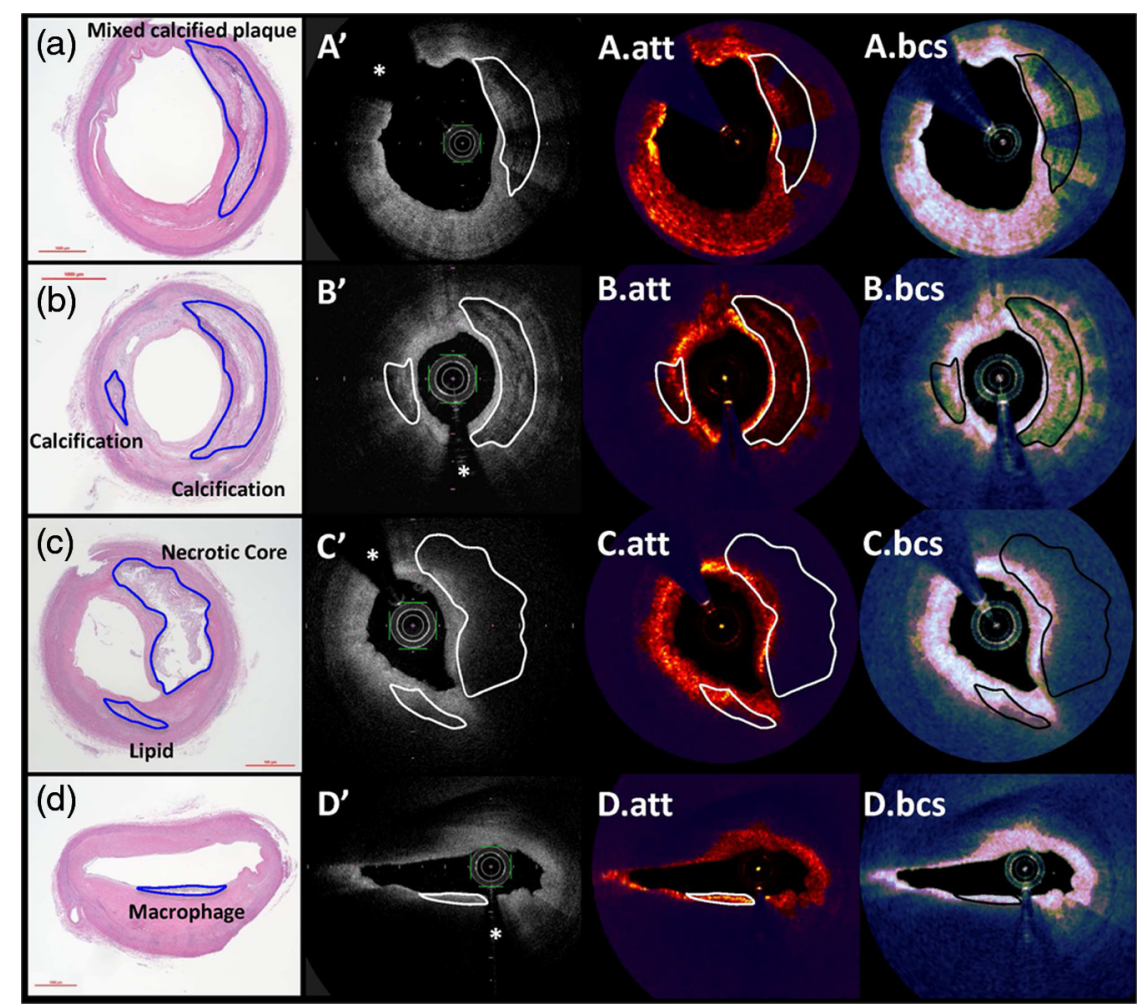

Fig. 1 (a)-(d) The histological cross sections and (a')-(d') the corresponding IVOCT cross sections. Star $\left({ }^{*}\right)$ marks the guide wire. The attenuation coefficients and the backscatter term are labeled as (a)-(d).att and (a)-(d).bcs, respectively. Noisy region behind the determined cut-off border is marked with dark blue overlay in attenuation and backscatter cross section. Plaques of interest are labeled in pathological cross sections, which have been aligned with other-related IVOCT images.

appearance in both images by two cardiologists (Y.S. and Y.O.). IVOCT cross sections with insufficient image quality and significant artifacts such as tangential artifacts were not used for the analysis. Based on the contours delineated by the pathologists in histology, the two cardiologists delineated the same tissue in matched IVOCT images in QCU-CMS (Quantitative Coronary Ultrasound-Clinical Measurement Systems, LKEB, Leiden University Medical Center, Leiden, The Netherlands) [Fig. 1(a')-1(d')]. When the border of the tissue could clearly be detected on IVOCT, the delineation followed the borderlines. When the border could not be clearly perceived, the contours in histological images were superimposed on the IVOCT images in order to yield a high reproducibility. If there were more than two layers of different ROIs in one cross section, only the most adluminal ROI was employed for the analysis considering the light property.

The two pathologists (G.N. and S.T.) were blinded to the IVOCT delineation and IVOCT light property analysis. At the stage of IVOCT delineation, to enable accurate colocalization of the histology with IVOCT images, two cardiologists (Y.S. and Y.O.) were unblinded to the histological identification. However, these cardiologists were blind to the following IVOCT light property analysis. The IVOCT light property analysis was performed independently from the either histological identification or the IVOCT delineation process.

\subsection{Implementation}

The experimental flow of analyzing the IVOCT values was designed as it is shown in Fig. 2.

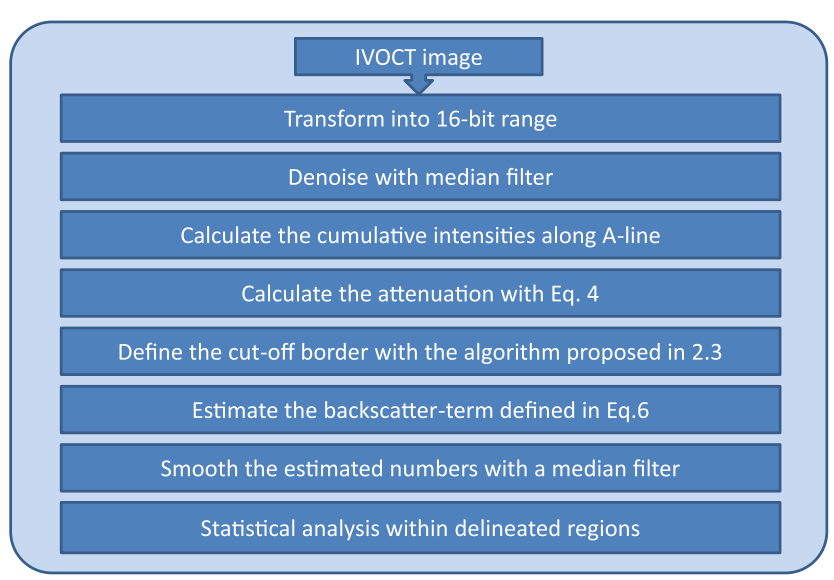

Fig. 2 The work flow for the experiment.

\subsubsection{Data transformation}

In the Terumo Lunawave imaging system, images are saved at 8bit depth to allow for a high system sensitivity, resulting into a lower dynamic range than those saved at 12- to 14-bit depth. ${ }^{40}$ The algorithm has been proposed based on a transition model that is linearly related to the detected light signal. Once the image is compressed nonlinearly, the estimated values can be in different ranges. As optical properties, it is expected that the estimated attenuation coefficient and backscatter term are in the same range for the same type of tissue rather than 
depending on the imaging systems. In order to overcome this issue, we used a matching look-up table to match 8-bit images generated with Lunawave from Terumo (Tokyo, Japan) to 16-bit range of images generated with Illumien Optis from St. Jude Medical (St. Paul, Minnesota). ${ }^{41}$

\subsubsection{Denoising}

A median filter with small window size is applied to the pixelwise DR estimated values to remove noise. Pixel-wise DR estimation allows statistical analysis within local regions. Although it might blur the edges between different types of tissues, the median filter will hardly affect the statistical distribution within the local region.

The median filter window size was chosen as $[3,11]$ for calculation of $\operatorname{cum} I_{b}$ and the same window size was applied for noise removal. With a pixel size of $5.4 \mu \mathrm{m}$, this length accordingly covers a range about $60 \mu \mathrm{m}$. The window size 11 is chosen to be the maximum odd number that covers approximately the thickness of small identifiable structures, e.g., a thin cap defined in the literature ${ }^{42}(<65 \mu \mathrm{m})$.

\subsection{Statistical Analysis}

The data were processed and analyzed with MATLAB. For each delineated region, the distribution of intensities, attenuation, and the backscatter term are analyzed. The mean, standard deviation (std), median, maximum (max), 95th percentile (95th per.), minimum (min), skewness, and kurtosis values were calculated within each delineated region. Values between different tissue types were compared with the two-sample $t$-test. Both threedimensional (3-D) scatter plots of the intensity, attenuation coefficient, and backscatter term and their principal component analysis (PCA) projected two-dimensional (2-D) scatter plot are generated.

\section{Results}

In MATLAB, the current code has been implemented with 3-D matrices calculation. Median filtering with a window size of 11 pixels on a PC with a $2.83-\mathrm{GHz}$ dual-core processor currently takes $7 \mathrm{~s}$ for every 100 IVOCT polar images of 512 A-lines with 900 depth points. The total time of calculating the attenuation coefficient and the backscatter term with cut-off border determination is $2.7 \mathrm{~min}$. Meanwhile as implemented in QCU-CMS, median filtering with a window size of 11 pixel points currently takes $24 \mathrm{~s}$ on a PC with a 2.66-GHz quad-core processor with hyperthreading for every 553 IVOCT polar images of 512 Alines with 1024 depth points. The total time of calculating the attenuation coefficient and the backscatter term without cut-off border determination is 23 and $22 \mathrm{~s}$, respectively. Currently, the processing time is reasonably fast and it is not a major concern. For the future clinical orientated on-the-fly application, the code and the implementation can be further optimized.

The statistical results are presented in Table 1 . The average and the standard deviation values were reported for all the regions from each tissue type, and the number of analyzed regions is given under the name of the tissue. The reported IVOCT intensity is the Terumo image intensity. Using the known calibration factor of the IVOCT images, the unit of the estimated attenuation coefficient is $\mathrm{mm}^{-1}$.

The comparison results of median values, mean values, standard deviation, maximum values, 95th percentile, minimum
Table 1 Statistical values.

\begin{tabular}{|c|c|c|c|c|}
\hline & & $\begin{array}{c}\text { Intensity } \\
\text { (no unit) } \\
\text { average } \pm \text { std }\end{array}$ & $\begin{array}{l}\text { Attenuation } \\
\left(\mathrm{mm}^{-1}\right) \\
\text { average } \pm \text { std }\end{array}$ & $\begin{array}{l}\text { Backscatter } \\
\text { (no unit) } \\
\text { average } \pm \text { std }\end{array}$ \\
\hline \multirow[t]{8}{*}{ Mixed } & Mean & $156.84 \pm 2.08$ & $1.68 \pm 0.14$ & $5.81 \pm 0.09$ \\
\hline & Median & $157.14 \pm 2.24$ & $1.44 \pm 0.17$ & $5.85 \pm 0.13$ \\
\hline & std & $26.00 \pm 0.59$ & $1.14 \pm 0.07$ & $0.71 \pm 0.03$ \\
\hline & Max & $236.64 \pm 3.04$ & $7.34 \pm 1.01$ & $7.56 \pm 0.19$ \\
\hline & 95th per. & $198.93 \pm 2.09$ & $3.89 \pm 0.25$ & $6.91 \pm 0.07$ \\
\hline & Min & $43.27 \pm 13.31$ & $0.11 \pm 0.06$ & $3.43 \pm 0.61$ \\
\hline & Skewness & $-0.15 \pm 0.07$ & $1.11 \pm 0.23$ & $-0.17 \pm 0.26$ \\
\hline & Kurtosis & $3.01 \pm 0.08$ & $4.38 \pm 0.94$ & $2.51 \pm 0.40$ \\
\hline \multirow[t]{8}{*}{ Cal } & Mean & $142.49 \pm 15.05$ & $0.87 \pm 0.24$ & $5.24 \pm 0.42$ \\
\hline & Median & $143.06 \pm 15.11$ & $0.68 \pm 0.16$ & $5.21 \pm 0.41$ \\
\hline & std & $26.43 \pm 2.19$ & $0.63 \pm 0.21$ & $0.65 \pm 0.07$ \\
\hline & Max & $232.68 \pm 16.39$ & $5.35 \pm 1.10$ & $7.37 \pm 0.29$ \\
\hline & 95th per. & $185.07 \pm 17.10$ & $2.17 \pm 0.73$ & $6.37 \pm 0.49$ \\
\hline & Min & $31.21 \pm 20.63$ & $0.13 \pm 0.03$ & $3.54 \pm 0.36$ \\
\hline & Skewness & $-0.11 \pm 0.12$ & $2.22 \pm 0.49$ & $0.24 \pm 0.21$ \\
\hline & Kurtosis & $3.03 \pm 0.16$ & $10.72 \pm 4.87$ & $2.78 \pm 0.29$ \\
\hline \multirow[t]{8}{*}{ Fib } & Mean & $172.17 \pm 10.39$ & $1.75 \pm 0.48$ & $6.22 \pm 0.32$ \\
\hline & Median & $173.93 \pm 10.42$ & $1.67 \pm 0.47$ & $6.24 \pm 0.34$ \\
\hline & std & $21.31 \pm 1.33$ & $0.61 \pm 0.17$ & $0.36 \pm 0.04$ \\
\hline & Max & $228.53 \pm 8.80$ & $4.55 \pm 1.20$ & $7.19 \pm 0.24$ \\
\hline & 95th per. & $204.07 \pm 9.49$ & $2.86 \pm 0.75$ & $6.78 \pm 0.30$ \\
\hline & Min & $73.77 \pm 19.97$ & $0.50 \pm 0.20$ & $5.06 \pm 0.35$ \\
\hline & Skewness & $-0.47 \pm 0.10$ & $0.79 \pm 0.36$ & $-0.24 \pm 0.35$ \\
\hline & Kurtosis & $3.29 \pm 0.26$ & $4.05 \pm 1.35$ & $3.01 \pm 0.60$ \\
\hline \multirow[t]{8}{*}{ Lip } & Mean & $160.40 \pm 3.27$ & $2.60 \pm 0.13$ & $6.23 \pm 0.11$ \\
\hline & Median & $161.60 \pm 3.78$ & $2.45 \pm 0.12$ & $6.27 \pm 0.10$ \\
\hline & std & $24.97 \pm 1.69$ & $1.16 \pm 0.08$ & $0.58 \pm 0.08$ \\
\hline & Max & $221.80 \pm 3.27$ & $6.47 \pm 0.58$ & $7.40 \pm 0.11$ \\
\hline & 95th per. & $199.80 \pm 1.92$ & $4.73 \pm 0.28$ & $7.06 \pm 0.08$ \\
\hline & Min & $64.00 \pm 15.43$ & $0.64 \pm 0.10$ & $4.60 \pm 0.28$ \\
\hline & Skewness & $-0.27 \pm 0.07$ & $0.56 \pm 0.10$ & $-0.35 \pm 0.15$ \\
\hline & Kurtosis & $2.82 \pm 0.15$ & $2.71 \pm 0.36$ & $2.41 \pm 0.43$ \\
\hline
\end{tabular}


Table 1 (Continued)

\begin{tabular}{|c|c|c|c|c|}
\hline & & $\begin{array}{c}\text { Intensity } \\
\text { (no unit) } \\
\text { average } \pm \text { std }\end{array}$ & $\begin{array}{l}\text { Attenuation } \\
\left(\mathrm{mm}^{-1}\right) \\
\text { average } \pm \text { std }\end{array}$ & $\begin{array}{l}\text { Backscatter } \\
\text { (no unit) } \\
\text { average } \pm \text { std }\end{array}$ \\
\hline \multirow[t]{8}{*}{ Mac } & Mean & $173.02 \pm 4.76$ & $3.41 \pm 0.38$ & $6.50 \pm 0.13$ \\
\hline & Median & $173.97 \pm 4.89$ & $3.22 \pm 0.45$ & $6.59 \pm 0.13$ \\
\hline & std & $24.63 \pm 3.91$ & $1.49 \pm 0.36$ & $0.57 \pm 0.15$ \\
\hline & Max & $232.05 \pm 6.70$ & $7.78 \pm 1.11$ & $7.57 \pm 0.18$ \\
\hline & 95th per. & $211.47 \pm 6.09$ & $6.05 \pm 0.85$ & $7.27 \pm 0.16$ \\
\hline & Min & $76.58 \pm 25.30$ & $0.62 \pm 0.35$ & $4.67 \pm 0.87$ \\
\hline & Skewness & $-0.28 \pm 0.14$ & $0.38 \pm 0.31$ & $-0.56 \pm 0.37$ \\
\hline & Kurtosis & $2.92 \pm 0.39$ & $2.53 \pm 0.42$ & $2.91 \pm 1.30$ \\
\hline \multirow[t]{8}{*}{$\mathrm{Nec}$} & Mean & $159.98 \pm 4.79$ & $1.89 \pm 0.54$ & $6.10 \pm 0.17$ \\
\hline & Median & $160.06 \pm 4.83$ & $1.72 \pm 0.44$ & $6.11 \pm 0.19$ \\
\hline & std & $22.43 \pm 2.06$ & $0.84 \pm 0.37$ & $0.51 \pm 0.10$ \\
\hline & Max & $228.28 \pm 10.94$ & $5.54 \pm 1.86$ & $7.44 \pm 0.25$ \\
\hline & 95th per. & $196.51 \pm 7.55$ & $3.51 \pm 1.26$ & $6.93 \pm 0.26$ \\
\hline & Min & $68.25 \pm 11.44$ & $0.42 \pm 0.12$ & $4.54 \pm 0.31$ \\
\hline & Skewness & $-0.10 \pm 0.20$ & $0.96 \pm 0.31$ & $-0.07 \pm 0.29$ \\
\hline & Kurtosis & $3.04 \pm 0.21$ & $4.09 \pm 1.22$ & $2.64 \pm 0.35$ \\
\hline
\end{tabular}

values, skewness, and kurtosis are shown in Figs. 3-10, respectively. The whisker box plot is given with six tissue groups. When the result of the two-sample $t$-test indicates the numbers are not significantly different, the two groups are marked with an X-mark. In the graph in the second row of each figure, the comparison of six tissues is shown as a relationship grid graph. For each paired tissue types, the square is colored as green if they are significantly different, red otherwise. On the right bottom panel, three grid graphs are combined with an "OR" operation; green indicates at least one square in the previous three graphs are marked as green, otherwise the square is marked as red. The 3-D scatter plots can be seen in Figs. 11 and 12. The PCA projected 2-D scatter plots are shown on the right-hand side of the top row for each figure.

In general, we found that the values within the delineated regions had a strong correlation with tissue types at the 5\% significance level by comparing the median, mean, std, 95th percentile, and minimum values of IVOCT intensity, estimated attenuation coefficient, and the backscatter term. This can be observed from the combined comparative graphs in Figs. 3 and 4 where the red color is not presented. Also from the PCA projected 2-D plot, the other values did not show clusters as distinguishable as they are in the mean and median values. The PCA projected 2-D plot of std values shows clear clusters of fibrous and the calcified plaques. Furthermore, it is worth noting that clear cluster patterns of the calcification, fibrous, and macrophages can be observed from the 2-D plot of the skewness values.

Most of the time, the three IVOCT numbers are complementary to each other for the differentiation of the six tissue types. For example, the median values (Fig. 3) of the attenuation are not significantly different between tissue types of mixed calcified (mixed) and the fibrous but the median values of intensities

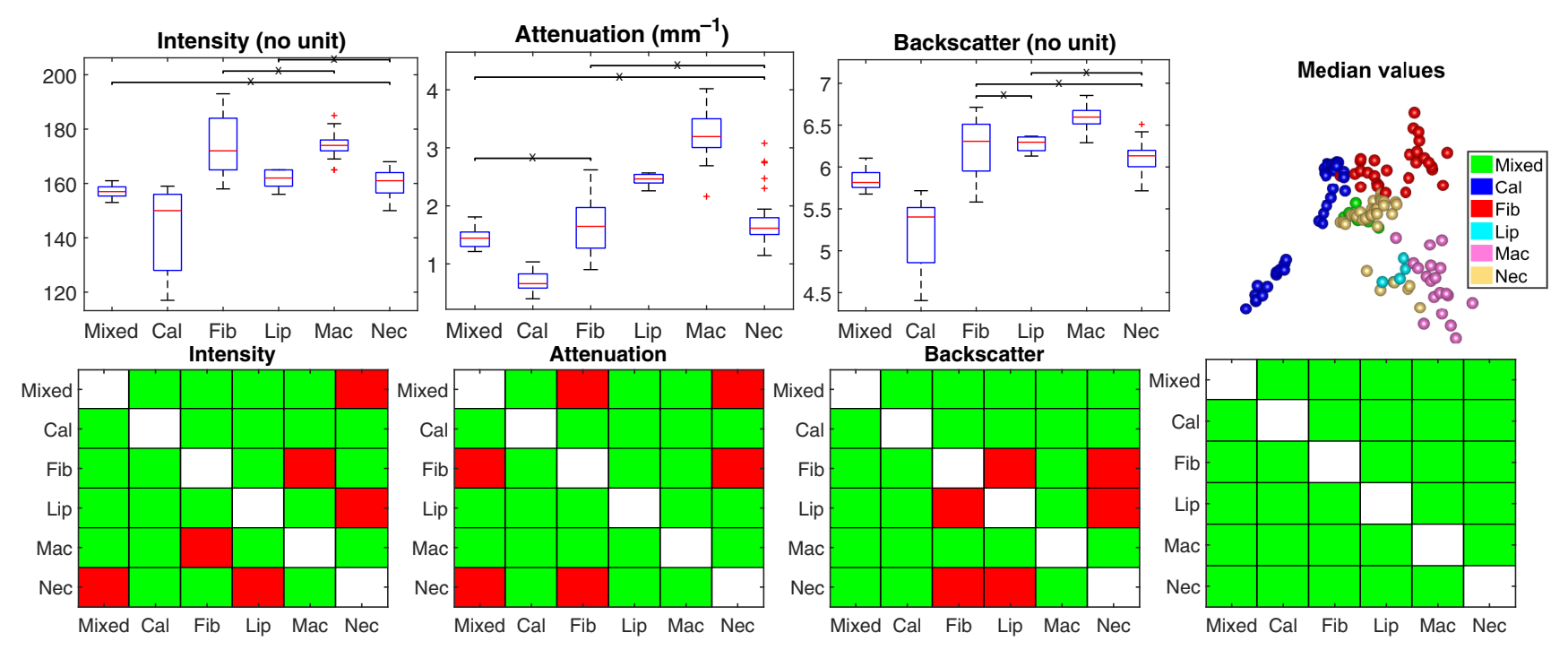

Fig. 3 Comparison of the median values of intensities (no unit), attenuation coefficient $\left(\mathrm{mm}^{-1}\right)$, and backscatter term (no unit) within each delineated region. The median values of the six types of tissue were compared to each other with two-sample $t$-test with significance $\alpha=0.05$. The whisker box plots can be seen on the top row, where paired tissue types that are not significantly different were connected with an " $X$ " mark. This statistical results are furthermore shown as a binary matrix, where the square is filled with green and gets a value "TRUE" if the relative tissue pair is statistically different, red and "FALSE" otherwise. Since it does not make sense to compare each tissue type to itself, the diagonal is filled with white. On the right-hand side at the bottom row, the three matrices are integrated with an "OR" operation. On the right-hand side of the top row, the PCA projected 2-D scatter plot is displayed. 

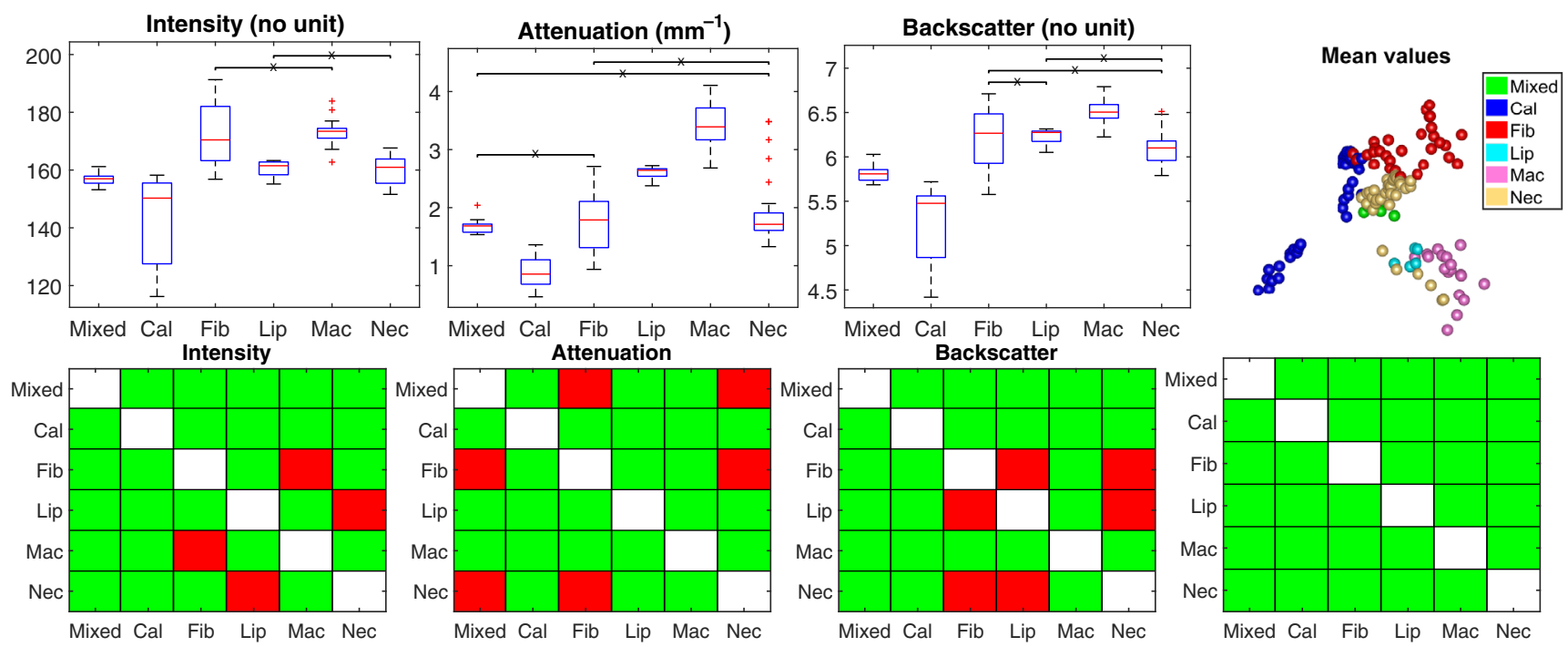

Fig. 4 Comparison of the mean values of intensities (no unit), attenuation coefficient $\left(\mathrm{mm}^{-1}\right)$, and backscatter term (no unit) within each delineated region. The layout information of this figure is the same as it is in Fig. 3.
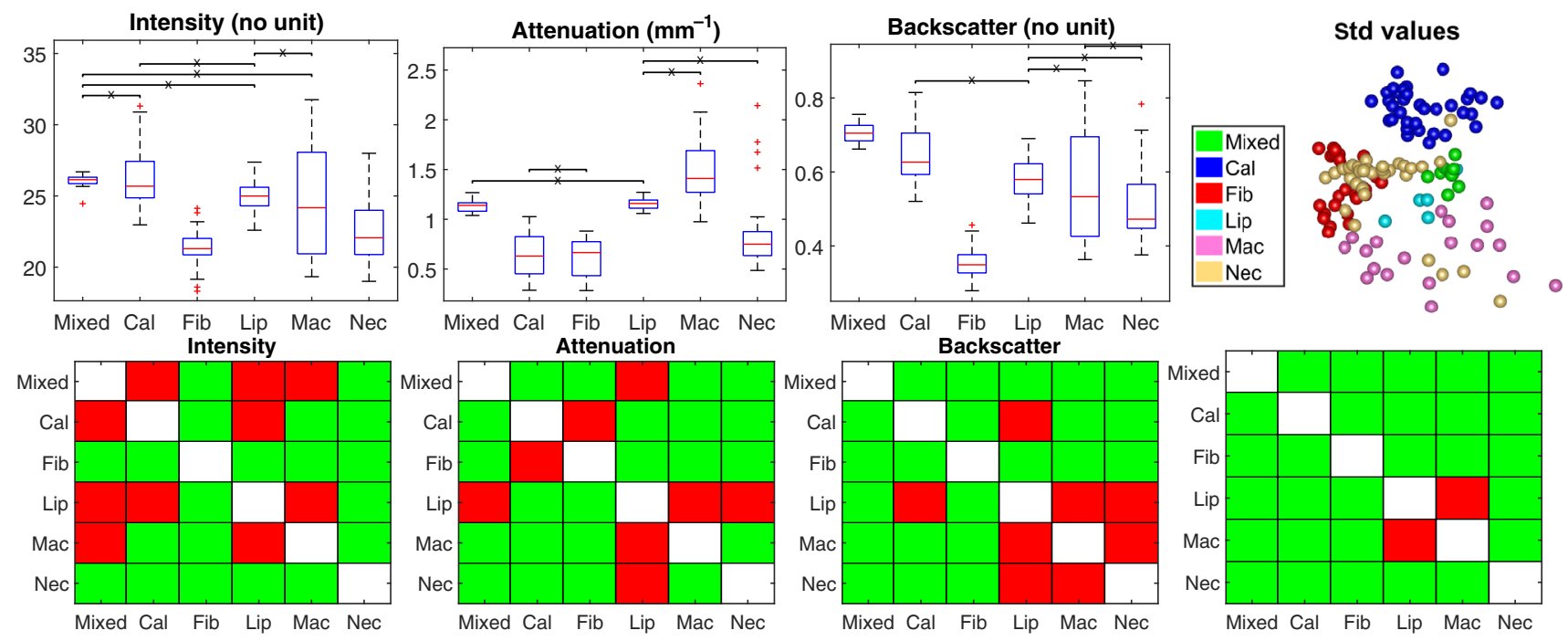

Fig. 5 Comparison of the standard deviations of intensities (no unit), attenuation coefficient $\left(\mathrm{mm}^{-1}\right)$, and backscatter term (no unit) within each delineated region. The layout information of this figure is the same as it is in Fig. 3.

and backscatter values are significantly different, and vice versa when comparing necrotic core to lipid-rich regions. This complement can be observed in all the compared figures (Figs. 3-9) but is most notable in median, mean, std, and maximum values (Figs. 3-6). In order to give an overview of the estimated values for the entire pullback, a "carpet view" image is generated. For a given stack of polar images, the maximum value for each A-line is determined in the first 1-mm depth below the lumen surface. In order to maintain the relative size of the plaques, the images are displayed with the lumen contour circumference normalized by the largest circumference over the entire pullback. The maximum projected attenuation and backscatter carpet views are shown in Figs. 13 and 14, where the largest circumference is $7.71 \mathrm{~mm}$. In order to show the values in a proper window level, key points of generating the color map are appointed both experimentally and referring to the work by Gnanadesigan et al. ${ }^{31}$ As it can be seen in Fig. 15, multiple pathological images show macrophage infiltration. The corresponding locations are marked as " $\mathrm{M}$ " in blue, and they are much brighter than other tissues in both carpet-views. This is consistent with our quantification analysis showing that both attenuation coefficient and the backscatter term are high. In order to show the estimated values for all six types of tissues, the attenuation coefficient and the backscatter term of IVOCT images in Fig. 1 are generated as well.

\section{Discussion}

This work aims at exploring the potential of DR attenuation coefficient, backscatter term, and image intensity in distinguishing 

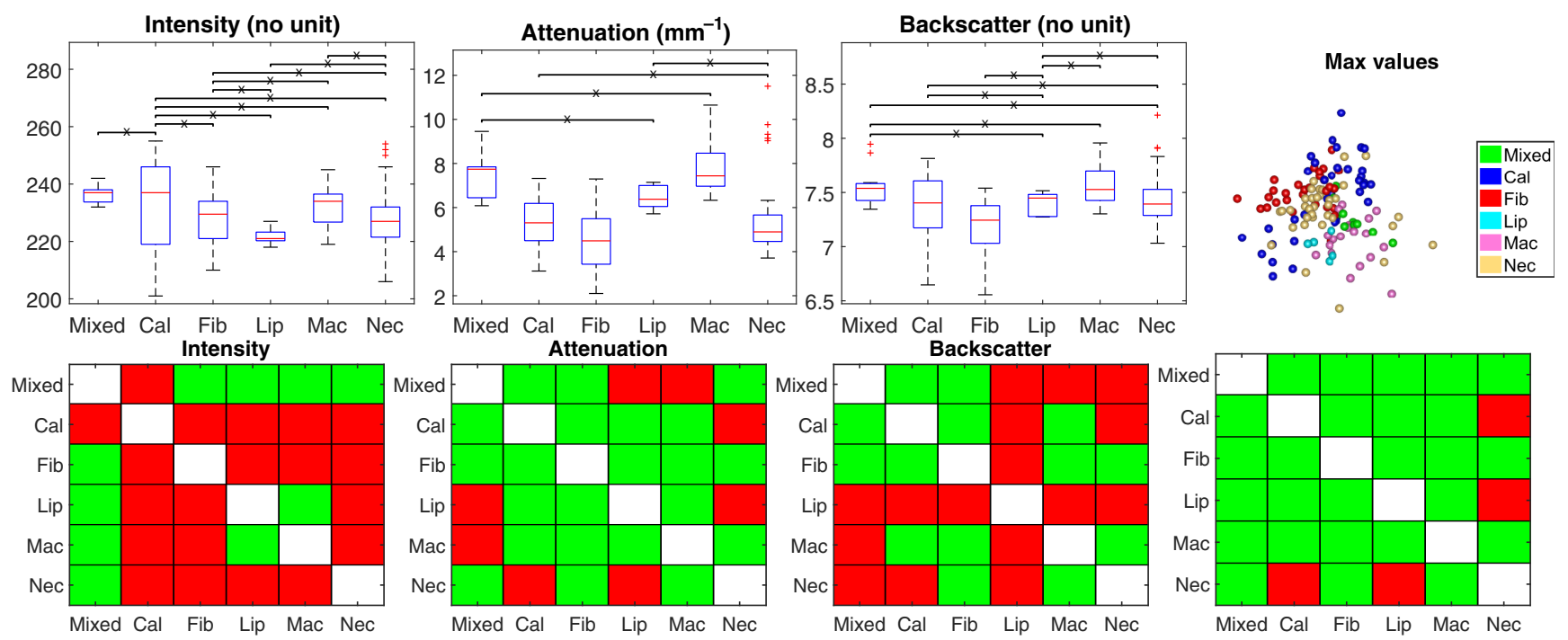

Fig. 6 Comparison of the maximum values of intensities (no unit), attenuation coefficient $\left(\mathrm{mm}^{-1}\right)$, and backscatter term (no unit) within each delineated region. The layout information of this figure is the same as it is in Fig. 3.
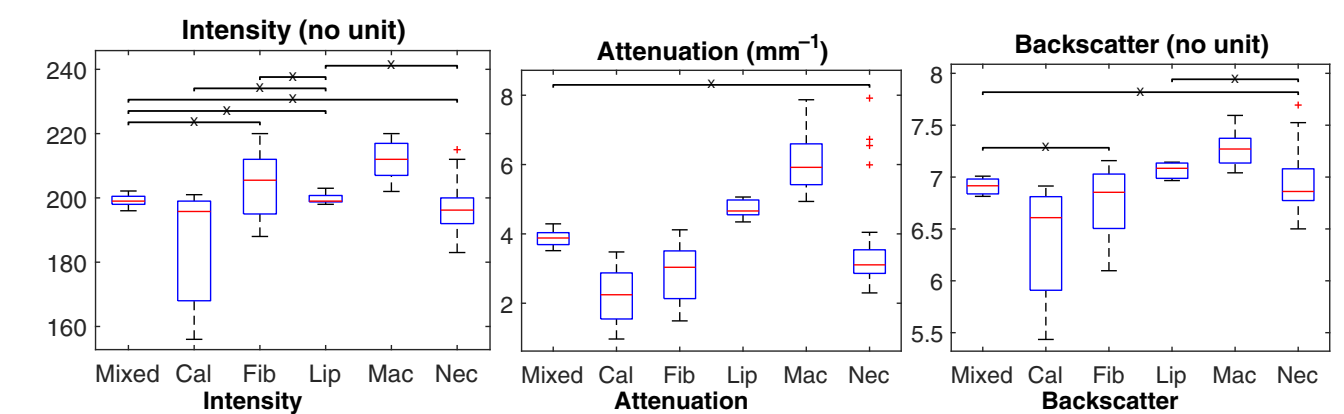

\section{5th percentle values}
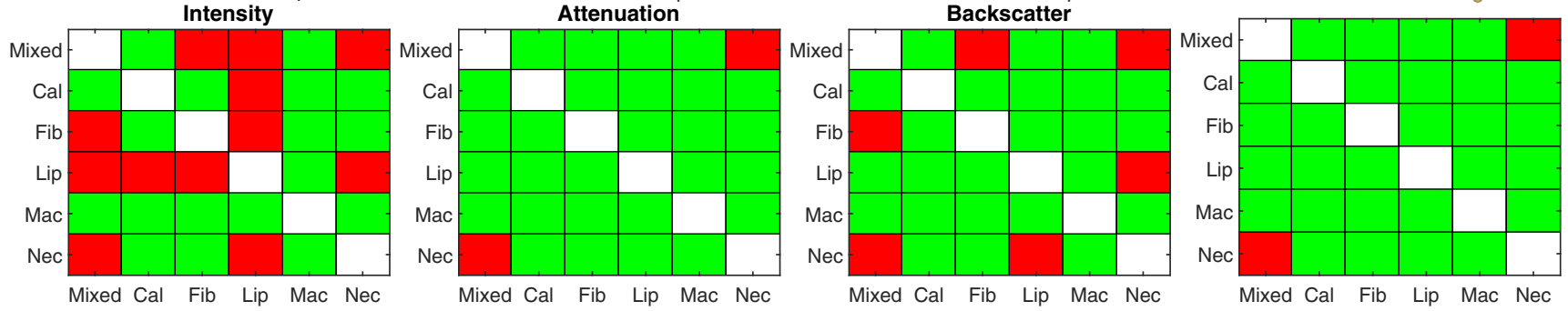

Fig. 7 Comparison of the 95th percentiles of intensities (no unit), attenuation coefficient $\left(\mathrm{mm}^{-1}\right)$, and backscatter term (no unit) within each delineated region. The layout information of this figure is the same as it is in Fig. 3.

different atherosclerotic tissue types with a robust implementation of the DR approach. We describe the implementation of the DR approach for the estimation of the attenuation coefficient in IVOCT images and extend it to estimate a backscatter term. To exclude the noisy regions, we describe an algorithm to automatically determine the cut-off border. Combining the estimated attenuation coefficient and the backscatter term with the image intensity, we report that it is possible to differentiate six types of clinically important tissues ${ }^{2,4,5}$ including the mixed calcified plaque, calcified plaque, fibrous plaque, lipid-rich plaque, plaque with macrophage, and plaque with necrotic core.

\subsection{Attenuation Coefficient}

The light transmission model of OCT signals was used as it has been introduced in the work of Vermeer et al. The attenuating term is modeled as $e^{-2 \mu_{t} r}$, while it has been modeled as $e^{-m_{t} r}$ in some CF approaches in the work of van Soest et al. The direct result of using this factor 2 in the exponential term is that the estimated attenuation coefficients are expected to be smaller than those previously reported in some of the studies. This difference affects only the absolute values rather than the comparative trend; therefore, we focus more on the comparative trend of the estimated values when comparing to the literature.

The attenuation coefficients of neointima have been reported as $0.35 \pm 1.38 \mathrm{~mm}^{-1}$ without lipid-laden and $2.00 \pm$ $1.16 \mathrm{~mm}^{-1}$ with lipid-laden in the work of Yonetsu et al. ${ }^{29}$ For the in-stent restenosis lesions of drug-eluting stents (DES) and bare-metal stents (BMS), the attenuation coefficients have been estimated as $1.69 \pm 0.84$ and $1.74 \pm 0.81 \mathrm{~mm}^{-1}$, respectively, in the study of Nagoshi et al. ${ }^{30}$ These values have been estimated with the LightLab software. Despite the estimation 


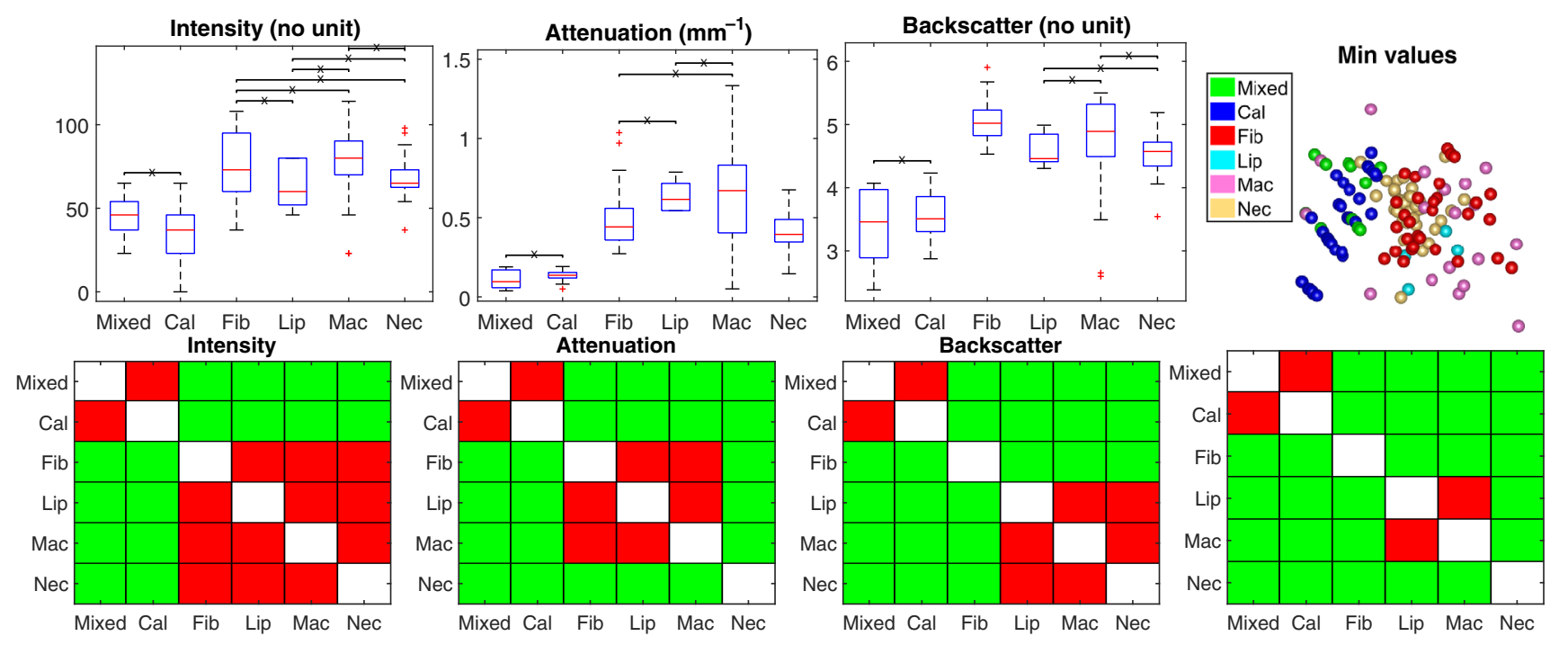

Fig. 8 Comparison of the minimum values of intensities (no unit), attenuation coefficient $\left(\mathrm{mm}^{-1}\right)$, and backscatter term (no unit) within each delineated region. The layout information of this figure is the same as it is in Fig. 3.
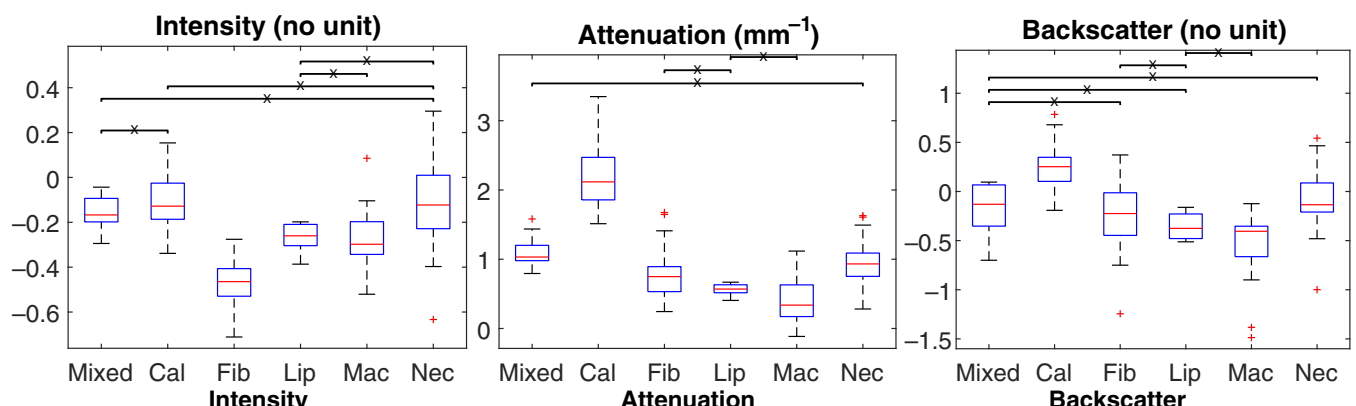

\section{Skewness values}
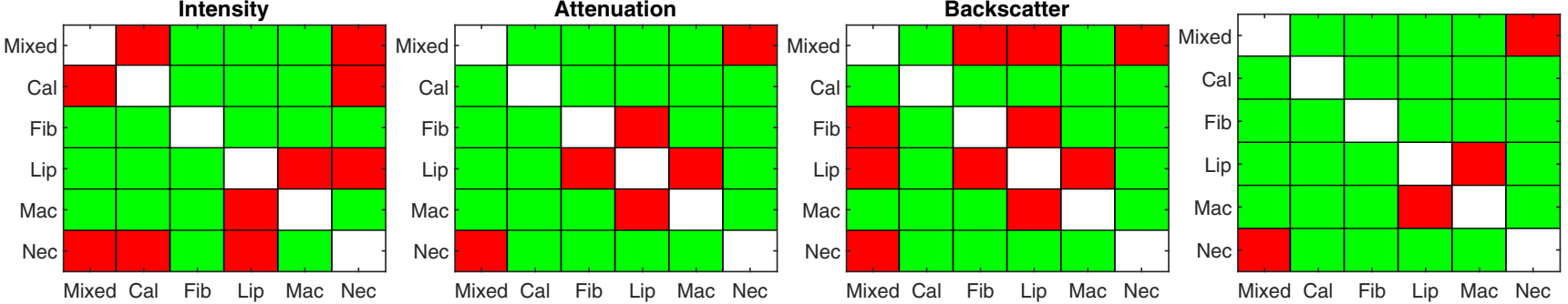

Fig. 9 Comparison of the skewness values of intensities (no unit), attenuation coefficient $\left(\mathrm{mm}^{-1}\right.$ ), and backscatter term (no unit) within each delineated region. The layout information of this figure is the same as it is in Fig. 3.

algorithm being unknown, these reported values are within the similar range of estimated attenuation coefficients of the fibrous tissue (mean: $1.75 \pm 0.48 \mathrm{~mm}^{-1}$, median: $1.67 \pm 0.47 \mathrm{~mm}^{-1}$ ). Taking the factor 2 into consideration, the estimated attenuation coefficient of the fibrous tissue (mean: $3.52 \pm 1.92 \mathrm{~mm}^{-1}$, median: $3.36 \pm 1.88 \mathrm{~mm}^{-1}$ ) is comparable to that reported as 2 to $5 \mathrm{~mm}^{-1}$ in the work of van Soest et al. ${ }^{9}$ and relatively lower than that reported as $6.4 \pm 1.2 \mathrm{~mm}^{-1}$ in the work of $\mathrm{Xu}$ et $\mathrm{al}^{8}$

For regions with macrophages infiltration, the range reported by van Soest et al. is $12 \mathrm{~mm}^{-1}$, and even can be as high as $15 \mathrm{~mm}^{-1}$ in a given example, which tends to be much higher than the other tissue types. Although we found attenuation coefficients of regions with calcification and macrophages much lower than what has been reported in these two studies, the comparative relationship of the values is the same, where the value of macrophages (mean: $3.41 \pm 0.38 \mathrm{~mm}^{-1}$, median: $\left.3.22 \pm 0.45 \mathrm{~mm}^{-1}\right)$ is the highest, and the value of the calcification (mean: $0.87 \pm 0.24 \mathrm{~mm}^{-1}$, median: $0.68 \pm 0.16 \mathrm{~mm}^{-1}$ ) is lower than that of fibrous tissue (mean: $1.75 \pm 0.48$, median: $1.67 \pm 0.47)$.

For the lipid-rich regions, we found a mean attenuation value of $2.60 \pm 0.13 \mathrm{~mm}^{-1}$ and median $2.45 \pm 0.12 \mathrm{~mm}^{-1}$. This is lower than what has been reported in the literature; $13.7 \pm 4.5^{8}$ and $>8.5^{31}$, but higher than fibrotic tissue which fits in the reported trends. A reason for this is that the cardiologists did draw the similar shape in IVOCT as was seen in the pathological slides (Fig. 1). Due to the high attenuation, the back border of the lipidic region is not seen in IVOCT and the deeper regions are dominated by noise. To avoid the inclusion of noise 

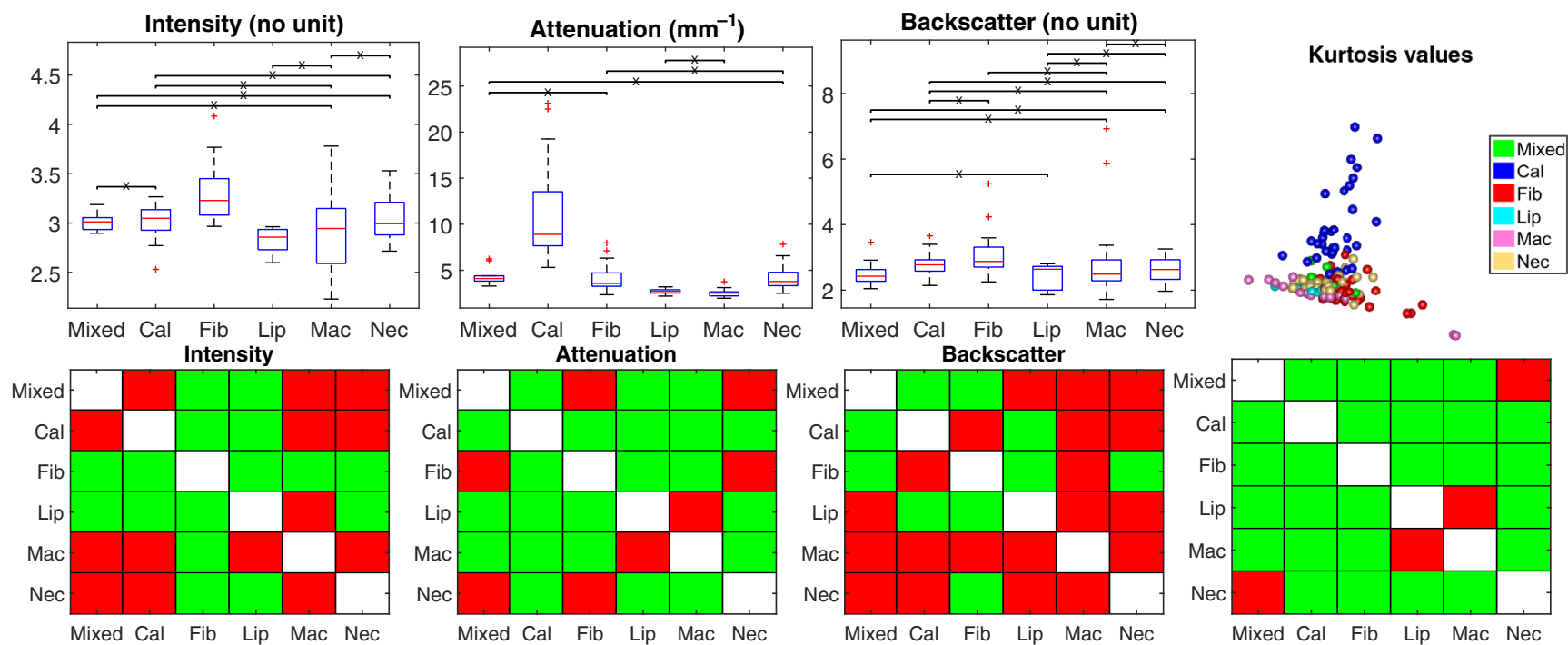

Fig. 10 Comparison of the kurtosis values of intensities (no unit), attenuation coefficient $\left(\mathrm{mm}^{-1}\right.$ ), and backscatter term (no unit) within each delineated region. The layout information of this figure is the same as it is in Fig. 3.
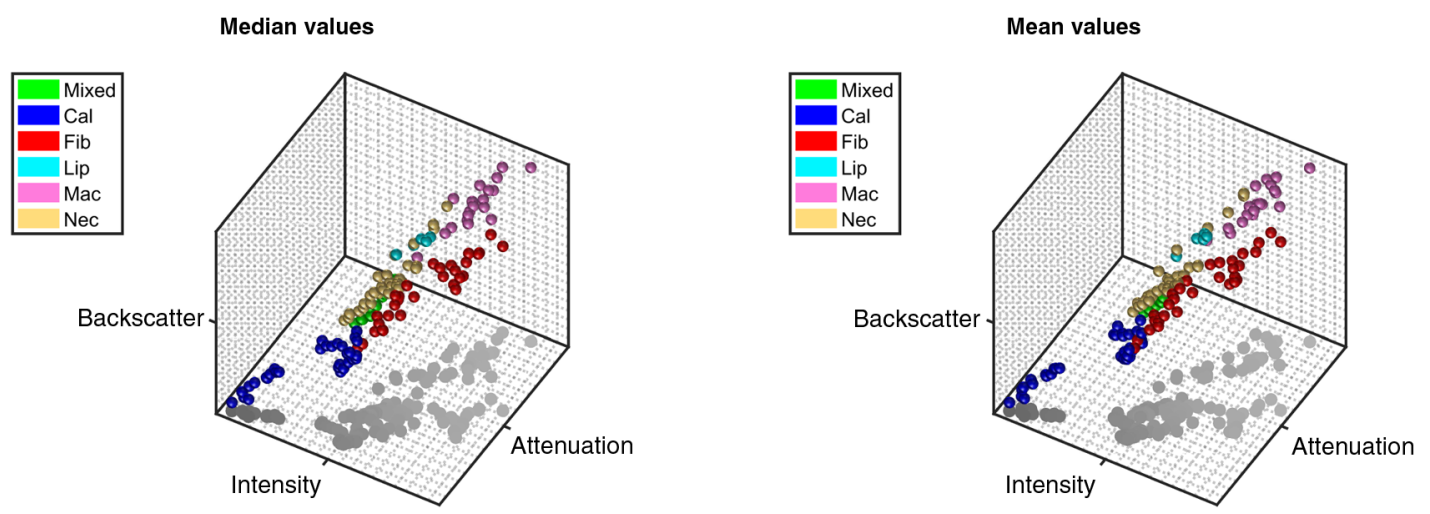

Std values
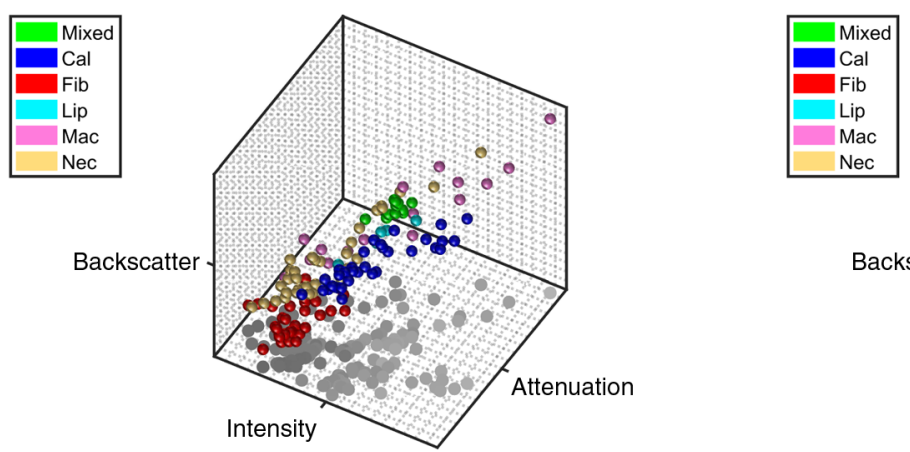

Max values

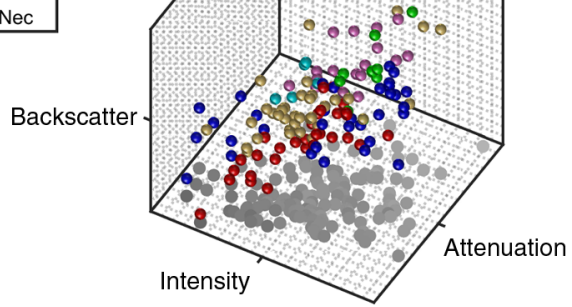

Fig. 11 3-D scatter plots of median (Video 1, MPEG, 8.1 MB [URL: http://dx.doi.org/10.1117/1.JBO.22.9 .096004.1]), mean (Video 2, MPEG, 8.1 MB [URL: http://dx.doi.org/10.1117/1.JBO.22.9.096004.2]), std (Video 3, MPEG, 7.8 MB [URL: http://dx.doi.org/10.1117/1.JBO.22.9.096004.3]), and maximum (Video 4, MPEG, 7.7 MB [URL: http://dx.doi.org/10.1117/1.JBO.22.9.096004.4]) values, which were normalized into $[0,1]$.

in the statistical parameters, we introduced the cut-off algorithm to remove the noisy regions. In order to compare relatively high values within the superficial limited regions of the lipid plaque, a higher percentile (95th) was considered for comparison as well.
It should be noted that the 95th percentile of the estimated values within the lipid-rich region can be as high as $4.73 \pm 0.28 \mathrm{~mm}^{-1}$ and that of macrophages can be $6.05 \pm 0.85 \mathrm{~mm}^{-1}$, which is much higher than that for other tissue types. The comparative 


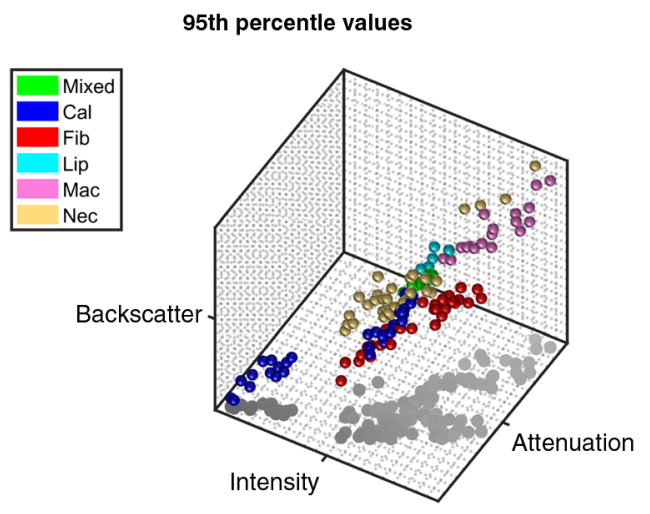

Skewness values

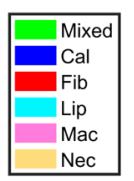

Backscatter

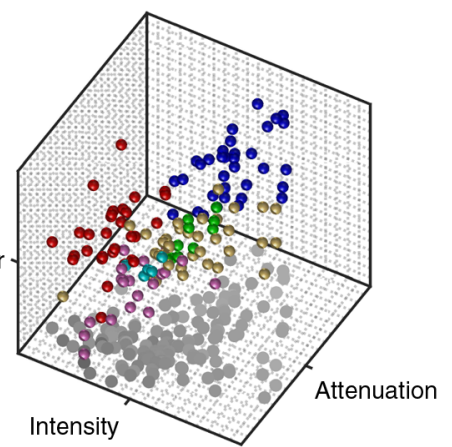

Min values

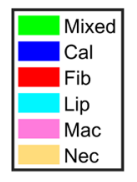

Backscatter

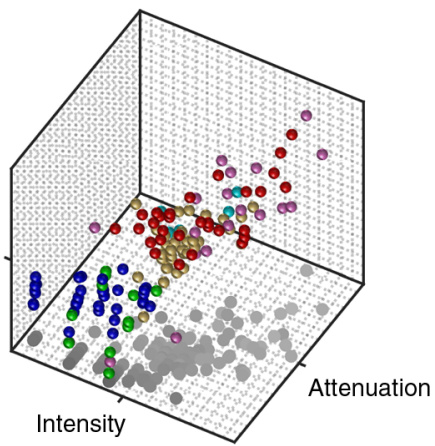

Kurtosis values

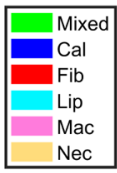

Backscatte

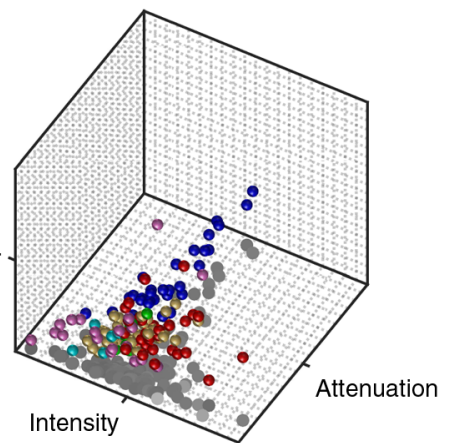

Fig. 12 3-D scatter plots of 95th percentile (Video 5, MPEG, 8.1 MB [URL: http://dx.doi.org/10.1117/1. JBO.22.9.096004.5]), minimum (Video 6, MPEG, 7.9 MB [URL: http://dx.doi.org/10.1117/1. JBO.22.9.096004.6]), skewness (Video 7, MPEG, 7.8 MB [URL: http://dx.doi.org/10.1117/1. JBO.22.9.096004.7]), and kurtosis values (Video 8, MPEG, 8.1 MB [URL: http://dx.doi.org/10.1117/1. JBO.22.9.096004.8]), which were normalized into $[0,1]$.

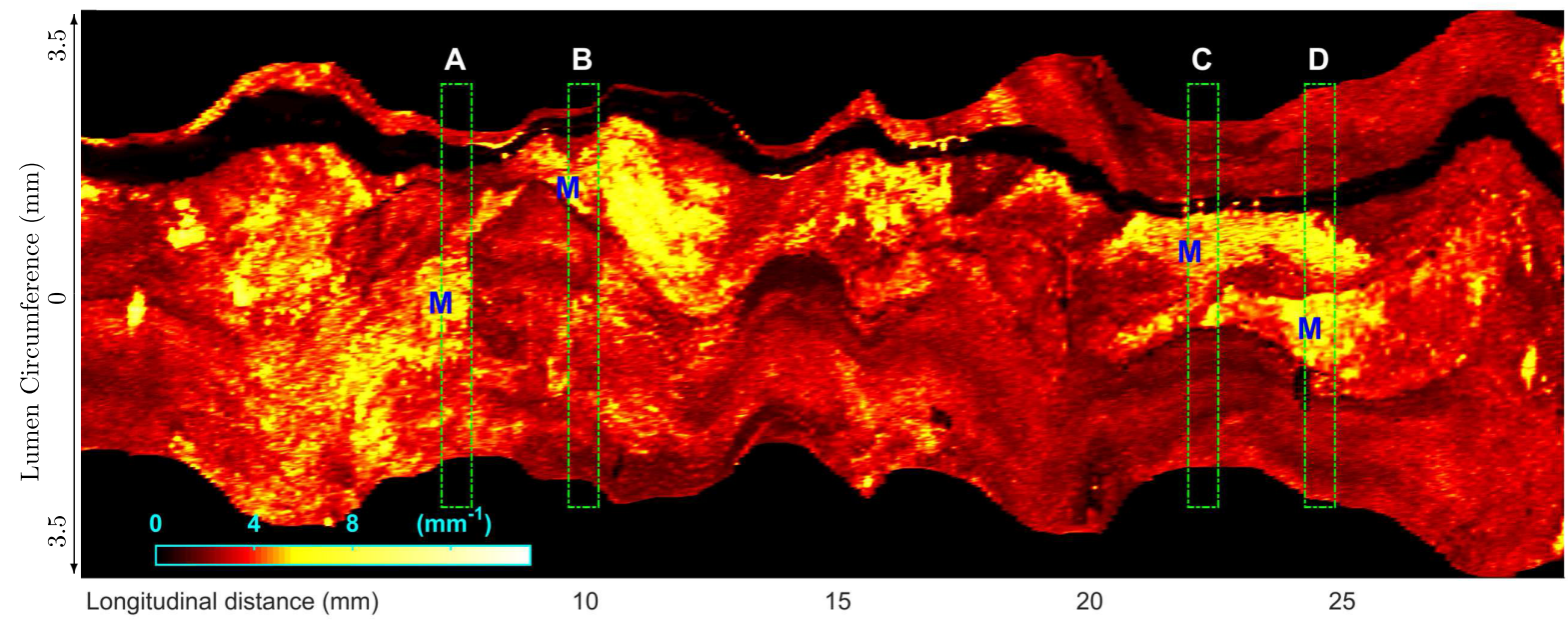

Fig. 13 The carpet view of the maximum attenuation coefficient within 1-mm depth. Four locations are marked with green squares and labeled as A, B, C, and D. "M" in blue marks the corresponding locations shown in the pathological images in Fig. 15, where macrophages are labeled. The cross sections of estimated attenuation coefficients can be seen in Fig. 15, labeled as "* att."

relationship in the mean, median, and 95th percentile values of the estimated attenuation coefficient is Cal $<$ Fib $<$ Lip $<$ Mac.

With respect to the necrotic tissue cores, since the plaques with macrophages and lipid were labeled separately, the necrotic cores chosen in this work are expected to have fewer macrophages infiltrations. This is shown in the estimated attenuation that the necrotic core (mean $1.89 \pm 0.54$, median $1.72 \pm 0.44$ ) is lower than lipid (mean $2.60 \pm 0.13$, median $2.45 \pm 0.12$ ) and macrophages (mean $3.41 \pm 0.38$, median $3.22 \pm 0.45$ ), and is 


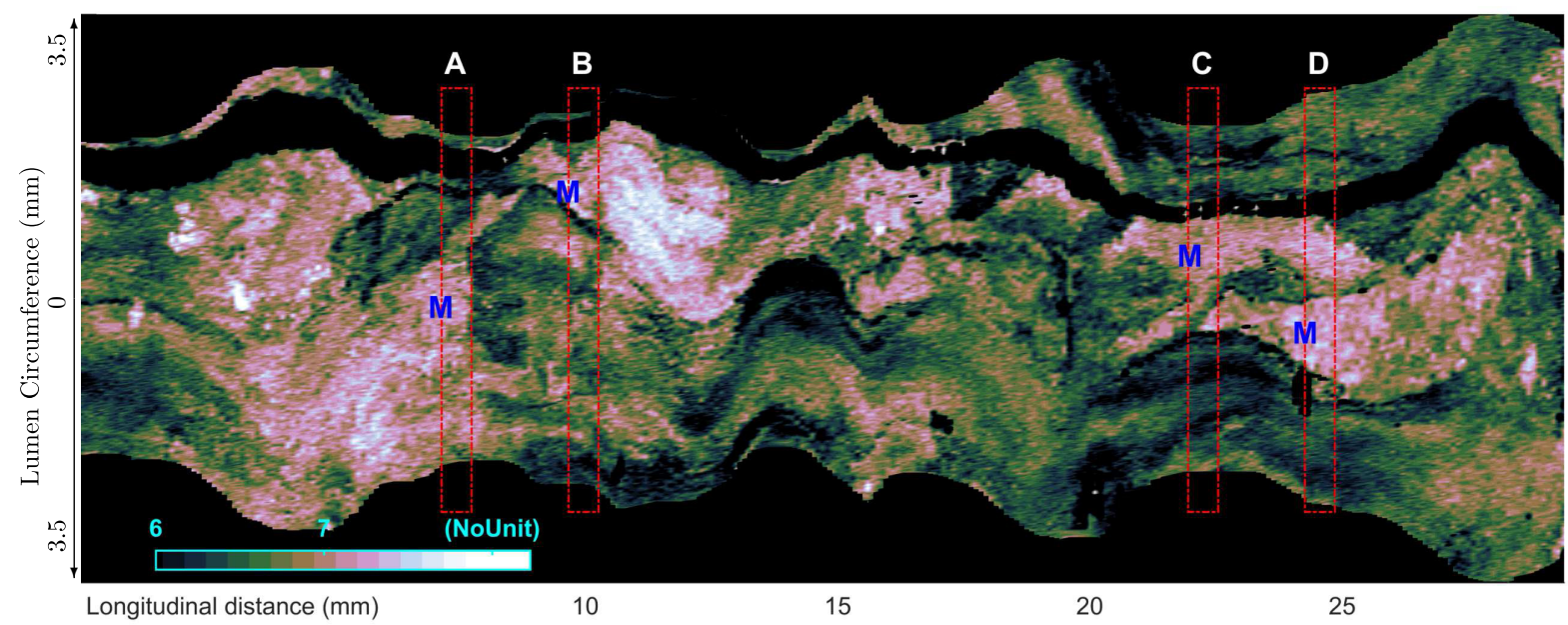

Fig. 14 The carpet view of the maximum backscatter term within 1-mm depth. Four locations are marked with red squares and labeled as A, B, C and D. "M" in blue marks the corresponding locations shown in the pathological images in Fig. 15, where macrophages are labeled. The cross sections of estimated backscatter term can be seen in Fig. 15, labeled as ".bcs."

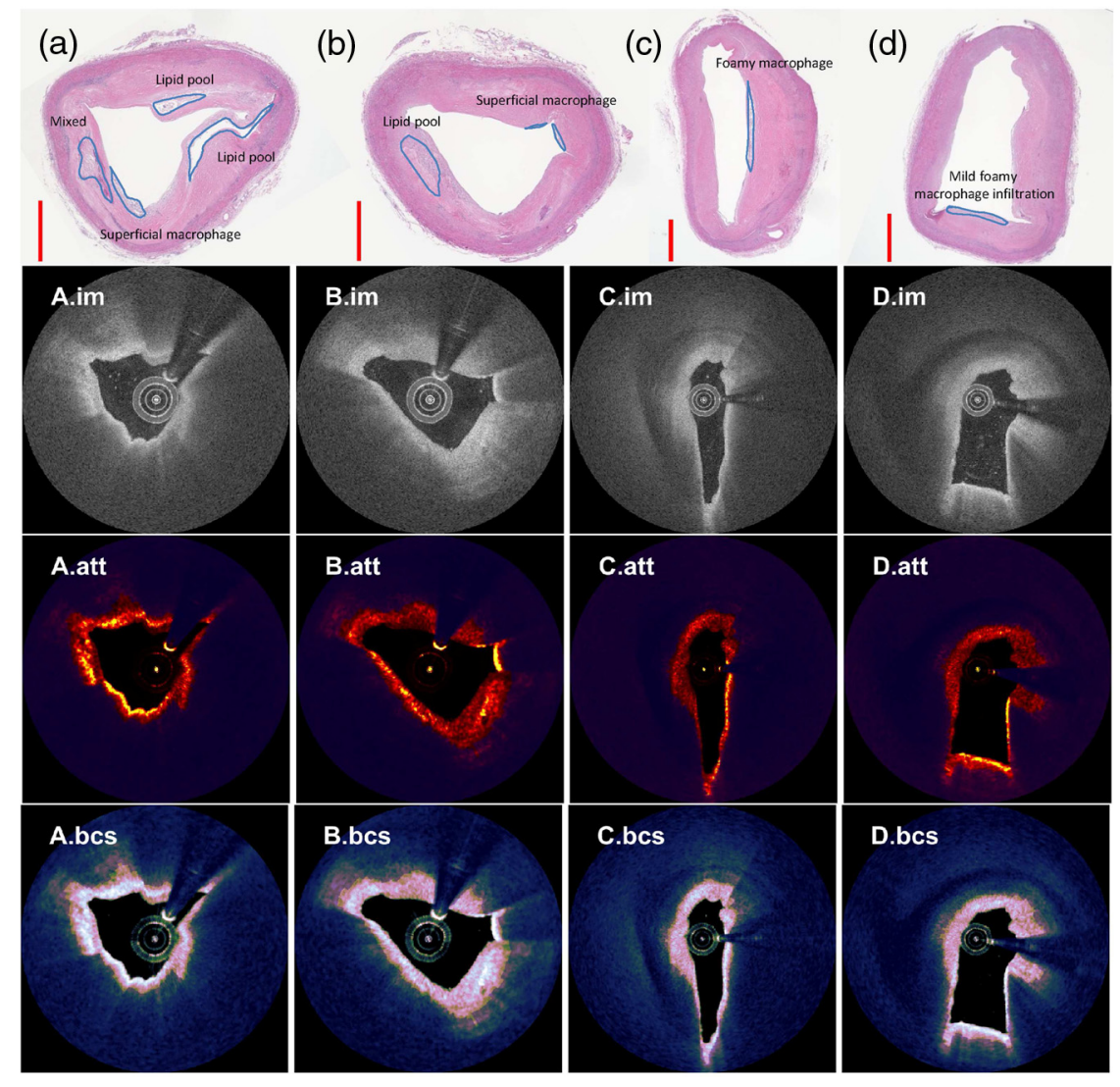

Fig. 15 The pathological cross sections, the IVOCT images ( ${ }^{*}$.im), the attenuation coefficients ( ${ }^{*}$.att), and the backscatter term (*.bcs) at four locations a, b, c, and d given in Figs. 13 and 14. Deeper region behind the determined cut-off border is marked with dark blue overlay in attenuation and backscatter cross section. Plaques of interest are labeled in pathological cross sections, which have been aligned with other IVOCT-related images.

just above the fibrotic tissue (mean $1.75 \pm 0.48$, median $1.67 \pm 0.47)$. In the work of van Soest, ${ }^{9}$ the necrotic core $\left(\geq 10 \mathrm{~mm}^{-1}\right)$ has been "read" as lipid plaque when compared to the study of $\mathrm{Xu}$ et al. We carefully inspected the regions in the IVOCT images, which were annotated as necrotic core by pathologists, but could not identify bright regions or spots as were shown in the work of van Soest thus resulting in a lower mean and median attenuation and backscatter. Therefore, the discrepancy compared to the work of van Soest is caused by a difference in the data. 
It should be noted that two clusters of values are observed in the median and mean values of the calcified plaque in the PCA projected 2-D plot (Fig. 3 and 4). This can be caused by the large variation in the median/mean values of the intensities (mean: $142.49 \pm 15.05$, median: $143.06 \pm 15.11)$ and the backscatter term (mean: $5.24 \pm 0.42$, median: $5.21 \pm 0.41$ ). However, it is worth noting that the distribution of the median/mean values in the attenuation is relatively condensed (mean: $0.87 \pm 0.24$, median: $0.68 \pm 0.16$ ), and the range of values is significantly different from any other tissue type.

\subsection{Backscatter Term}

As it has been stated, the DR attenuation was estimated with the precondition that the attenuation and the backscatter coefficients are linearly related to each other. Rather than estimating the backscatter coefficient, the proposed calculation of the backscatter term in our work is based on the principle that the "offset" of the linear fitting is the backscatter-related term, the exponential of which has been shown to be linearly related to the actual backscatter coefficient in the work of Schmitt et al. ${ }^{16}$ Applying this principle, the DR model was extended to estimate this term pixel wise. Our analysis of the optical model shows that this term is related to both the backscatter coefficient and the initial power of the incident light. Results show that this term provides different comparative graphs and additional information for tissue differentiation. For example, fibrotic tissue was not significantly different from macrophages for the mean intensities, but they are statistically different in mean values of the estimated backscatter term (Fig. 4).

To the best of our knowledge, only three studies have reported the backscatter coefficient in the literature of tissue analysis within vessel walls. ${ }^{8,29,30}$ The estimated values with the LightLab software have been reported for neointima as $6.19 \pm 0.81$ without lipid-laden and $6.56 \pm 0.60$ with lipidladen, ${ }^{29}$ and have been reported as $6.92 \pm 0.42$ and $7.14 \pm 0.41$ for the in-stent restenosis lesions of DES and BMS, respectively. ${ }^{30}$ This range is comparable to that of our estimated values of the fibrous region (mean: $6.22 \pm 0.32 \mathrm{~mm}^{-1}$, median: $6.24 \pm 0.34 \mathrm{~mm}^{-1}$ ).

The backscatter coefficient has been measured by Xu et al. ${ }^{8}$ as $18.6 \pm 6.4 \mathrm{~mm}^{-1}$ for fibrous tissue, $28.1 \pm 8.9 \mathrm{~mm}^{-1}$ for lipid-rich tissue, and $4.9 \pm 1.5 \mathrm{~mm}^{-1}$ for the calcified plaque. Although the absolute values are not comparable to our values due to different experimental settings and a fundamentally different approach for the estimation as mentioned before, we still observe similar patterns. The fibrous plaque has been reported to have "high backscatter and low attenuation," while our results do show that backscatter for fibrotic tissue is relatively high with relatively low attenuation. When comparing fibrous regions to macrophage regions, it is worth noting that the mean, median, and the 95th percentile values for the former are lower, suggesting a more compact distribution. This may relate to the speckle pattern caused by the inhomogeneous distribution of the foam cells. The estimated value in calcified regions (mean: $5.24 \pm 0.42$, median: $5.21 \pm 0.41)$ is significantly lower than that in fibrous regions (mean: $6.22 \pm 0.32$, median: $6.24 \pm 0.34)$. The lipid is not significantly different from fibrous regions in the mean and median of the estimated backscatter term, but the prior is higher than the latter in the 95th percentile values (95th percentile: $7.06 \pm 0.08$ versus $6.78 \pm 0.30$ ).

\subsection{IVOCT Intensity}

Most studies in the literature focused on the comparisons of only the attenuation coefficient, ${ }^{9,20-24,28,32}$ or the IVOCT intensities, ${ }^{6,7}$ while a few studies combined the attenuation and the backscatter coefficients. ${ }^{8,29,30}$ The two-sample $t$-test results suggest that the IVOCT image intensity does additionally contribute to the differentiation of the tissue types. For example, for the fibrotic versus necrotic tissue, the mean values of neither the attenuation nor the backscatter term are significantly different (Fig. 7), while the mean values of the intensities are significantly different.

On the other hand, it has been found in our previous work that the IVOCT intensities can be affected by the eccentric position of the catheter when light travels a long distance through the flush medium or enters the arterial wall with a large incident angle. ${ }^{43}$ In this analysis, this bias on intensities has been minimized by using only the lesions with front edges that are almost perpendicular to the incident light from the catheter. However, it is usually difficult to avoid this bias in clinical data. It has been shown that the intensities can be normalized with the summation of the total amount of incident light, yet additional statistical analysis on healthy tissues is required. From the principle equations for estimating attenuation coefficient and backscatter term, one may note that these two optical parameters are normalized with the summation of the total amount of intensities, and thus are expected to be less affected by this particular bias. Therefore, practical analysis with in vivo data is suggested to be focused more on these two estimated optical properties.

\subsection{Limitations}

The catheter-related terms were not considered in this work. As far as we know, even in the most recently published studies in the literature of the experimental optical measurements, the catheter-related term was reported to be calibrated either with OCT measurements in a weakly scattering media, ${ }^{19}$ or a particular phantom. ${ }^{44}$ Since we had access only to the image data and not the used catheter, the catheter specific calibration could not be done. However, as it has been discussed in the work of Vermeer et al., the maximum bias caused by the confocal function to the estimated DR attenuation is $\pm 1 /\left(4 z_{R}\right)$ at depth $z=z_{0} \pm 2 z_{R}$ where $z_{0}$ is the focus position and the $z_{R}$ is the Rayleigh length. Calculating with $z_{R}=2 \mathrm{~mm}$ used in the work of Ughi et al., ${ }^{32}$ the maximum bias is expected to be around $\pm 0.125 \mathrm{~mm}^{-1}$, which is relatively small compared to the scale for the estimated DR attenuation coefficient with IVOCT images.

Another potential issue could be that different tissue types have different refractive indices. This is not taken into account during the reconstruction process. This could lead to a difference in image resolution in different tissue types, while a fixed resolution is used. Ideally, different refractive indices should be applied for different types of tissues during the reconstruction. However, it requires prior knowledge of tissue compositions, which is almost impossible in practice.

When the resolution term is modeled as a function related to depth and tissue type, the impact on the accuracy of estimated optical coefficients can vary. Equation (4) will become

$\mu[i, j] \approx \frac{I[i, j]}{2 \sum_{y=j+1}^{\infty} \Delta[i, y \mid X] \cdot I[i, y]}$, 
$X$ is a certain tissue type, $\Delta[i, y \mid X]$ is the pixel size at location $[i, y]$. The pixel size is inversely proportional to the refractive index. As it has been reported in the literature, ${ }^{8}$ the refractive indices of fibrous tissue, lipid pool, and calcification tend to be around $1.35,1.43$, and 1.50 with a center wavelength of $1320 \mathrm{~nm}$, thus the pixel size in the previous two types of tissue should be larger than $5.4 \mu \mathrm{m}$, while calcified lesions yield a smaller pixel size. Then, it is expected that estimated values can be maximally overestimated by a factor of $1.447 / 1.35$ in normal/fibrotic tissue or underestimated by a factor of $1.447 /$ 1.50 when a large calcified lesion is present. Taking macrophages in this study as an example, the median value of estimated attenuation is $3.22 \mu \mathrm{m}$. When the pixel size is considered to be the only factor that affects the estimation, the real value is expected to be within an interval of [3.00, 3.34]. Given the large difference in attenuation to the other types of tissues, the impact caused by the imaging resolution in detecting the presence of macrophages is expected to be small.

For the sake of real-time computing, a median filter is applied rather than a more complex speckle noise removal filter such as the iwhTV denoising published recently ${ }^{34}$ or the entropy filter used by Jimenez et al. ${ }^{45}$ A fixed window size has been selected as around $60 \mu \mathrm{m}$. As it was discussed, the median filter blurs only the attenuation at the border of different tissues, and it affects the local statistical numbers minimally.

The statistical numbers for different tissue types have been calculated within the manually delineated regions. Other tissue types such as loose connective tissue, proteoglycans, calcified nodules, and smooth muscle cell-rich fibrous tissue were not assessed in this study because of the limited study sample size and the absence of specific staining. ${ }^{42}$ Artifacts would inevitably affect the light property analysis. These points can be limitations in the future for tissue classification and subjects for a further investigation.

\subsection{Future Directions}

Generating a distribution map indicating the probability of each type of plaque is one of the interesting directions in future work. Toward this goal as it has been proposed in the literature, ${ }^{26}$ the following works need to be done. Enough features should be extracted, including the first- and second-order statistical features $^{27}$ of intensities, attenuation coefficient, backscatter term, the power spectrum parameters, etc. Feature selection should be used to remove strongly linear-related features, which can result in a noninvertible covariance matrix. Then, a likelihood density map can be generated for each type of tissue. For a fair comparison between tissue types to determine the most likely tissue type for each pixel, the prior probabilities are necessary to be obtained in advance. Usually, manually reviewing a pullback series is time-consuming and points of interest easily cannot be overlooked due to the huge amount of data (up to over 1000 images). Integrated with the automated lumen detection in QCU-CMS ${ }^{46}$ carpet views as shown in Figs. 13 and 14 can be generated robust and fast without the need of predefining the segmentation of the lumen tissue. This overview of the examined coronary vessel wall can potentially improve the abnormality determination and further help with the treatment decision ${ }^{47,48}$ for coronary diseases, e.g., for stent placement and risk assessment of the patient in an automated way which is lacking in the current clinical practice.

\section{Conclusion}

In order to differentiate atherosclerotic tissue with IVOCT images, the DR approach has been implemented in the IVOCT images to robustly estimate the attenuation coefficient and the backscatter term. A cut-off algorithm has been proposed to efficiently exclude noisy regions. The reported values for each type of tissue are consistent with the findings in the literature. Despite assumptions made in the DR approach, statistical analysis shows that the estimated attenuation coefficient and the backscatter term can differentiate six tissue types in the lumen vessel; mixed calcified plaque, fibrous, calcification, lipid, macrophages, and necrotic core in combination with the image intensity.

\section{Disclosures}

Dr. Onuma reports to be a member of the advisory board of Abbott Vascular; Professor Serruys reports personal fees from Abbott, AstraZeneca, Biotronik, Cardialysis, from GLG Research, Medtronic, Sinomedical Sciences Technology, Socit Europa Digital Publishing, Stentys France, Svelte Medical Systems, Volcano, and St. Jude Medical, outside the submitted work.

\section{Acknowledgments}

This research was supported by the China Scholarship Council (No. 201206130062).

\section{References}

1. V. Tuchin, "Optical coherence tomography and heterodyning imaging," Chapter 9 in Tissue Optical Light Scattering Methods Instruments Medical Diagnosis, 2nd ed., pp. 565-589, SPIE Press, Bellingham, Washington (2007).

2. F. Prati et al., "Expert review document on methodology, terminology, and clinical applications of optical coherence tomography: physical principles, methodology of image acquisition, and clinical application for assessment of coronary arteries and atherosclerosis," Eur. Heart J. 31(4), 401-415 (2010).

3. D. Mozaffarian et al., "Heart disease and stroke statistics-2016 update," Circulation 133(4), e38-e360 (2016).

4. G. J. Tearney et al., "Consensus standards for acquisition, measurement, and reporting of intravascular optical coherence tomography studies: a report from the international working group for intravascular optical coherence tomography standardization and validation," J. Am. Coll. Cardiol. 59(12), 1058-1072 (2012).

5. E. Tenekecioglu et al., "Intracoronary optical coherence tomography: clinical and research applications and intravascular imaging software overview," Cathet. Cardiovasc. Intervent. 89(4), 679-689 (2017).

6. S. Nakatani et al., "Temporal evolution of strut light intensity after implantation of bioresorbable polymeric intracoronary scaffolds in the absorb cohort b trial," Circ. J. 78(8), 1873-1881 (2014).

7. S. Nakatani et al., "Bioresorption and vessel wall integration of a fully bioresorbable polymeric everolimus-eluting scaffold: optical coherence tomography, intravascular ultrasound, and histological study in a porcine model with 4-year follow-up," JACC: Cardiovasc. Interventions $\mathbf{9}$, 838-851 (2016).

8. C. Xu et al., "Characterization of atherosclerosis plaques by measuring both backscattering and attenuation coefficients in optical coherence tomography," J. Biomed. Opt. 13(3), 034003 (2008).

9. G. van Soest et al., "Atherosclerotic tissue characterization in vivo by optical coherence tomography attenuation imaging," J. Biomed. Opt. 15(1), 011105 (2010).

10. K. A. Vermeer et al., "Depth-resolved model-based reconstruction of attenuation coefficients in optical coherence tomography," Biomed. Opt. Express 5(1), 322-337 (2014). 
11. G. J. Tearney, "CT imaging of macrophages: a bright spot in the study of inflammation in human atherosclerosis," JACC: Cardiovasc. Imaging 8(1), 73-75 (2015).

12. J. E. Phipps et al., "Macrophages and intravascular OCT bright spots: a quantitative study," JACC: Cardiovasc. Imaging 8(1), 63-72 (2015).

13. Y. Minami et al., "Lipid-lowering therapy stabilizes the complexity of non-culprit plaques in human coronary artery: a quantitative assessment using OCT bright spot algorithm," Int. J. Cardiovasc. Imaging 33(4), 453-461 (2017).

14. M. Villiger et al., "Intravascular polarization sensitive optical coherence tomography in human patients," in Conf. on Lasers and Electro-Optics, Conf. on Lasers and Electro-Optics, AW1O.2, Optical Society of America (2016)

15. J. Li et al., "Polarization sensitive optical frequency domain imaging system for endobronchial imaging," Opt. Express 23, 3390-3402 (2015).

16. J. M. Schmitt et al., "Measurement of optical properties of biological tissues by low-coherence reflectometry," Appl. Opt. 32(30), 60326042 (1993).

17. J. M. Schmitt et al., "Optical-coherence tomography of a dense tissue: statistics of attenuation and backscattering," Phys. Med. Biol. 39(10), 1705-1720 (1994).

18. E. A. Sergeeva et al., "Novel algorithm of processing optical coherence tomography images for differentiation of biological tissue pathologies," J. Biomed. Opt. 10(6), 064024 (2005).

19. M. Almasian and D. J. Faber, "Validation of quantitative attenuation and backscattering coefficient measurements by optical coherence tomography in the concentration- dependent and multiple scattering regime," J. Biomed. Opt. 20(12), 121314 (2015).

20. D. Faber et al., "Quantitative measurement of attenuation coefficients of weakly scattering media using optical coherence tomography," Opt. Express 12(19), 4353-4365 (2004).

21. P. Lee, W. Gao, and X. Zhang, "Performance of single-scattering model versus multiple-scattering model in the determination of optical properties of biological tissue with optical coherence tomography," Appl. Opt. 49(18), 3538-3544 (2010).

22. E. C. C. Cauberg et al., "Quantitative measurement of attenuation coefficients of bladder biopsies using optical coherence tomography for grading urothelial carcinoma of the bladder," J. Biomed. Opt. 15(6), 066013 (2010).

23. P. Gong et al., "Assessment of human burn scars with optical coherence tomography by imaging the attenuation coefficient of tissue after vascular masking," J. Biomed. Opt. 19(2), 021111 (2014).

24. J. Li et al., "Quantitative measurement of optical attenuation coefficients of cell lines cne1, cne2, and np69 using optical coherence tomography," Lasers Med. Sci. 28(2), 621-625 (2013).

25. Y. Yang et al., "Optical scattering coefficient estimated by optical coherence tomography correlates with collagen content in ovarian tissue," J. Biomed. Opt. 16(9), 090504 (2011).

26. M. Mujat et al., "Automated algorithm for breast tissue differentiation in optical coherence tomography," J. Biomed. Opt. 14(3), 034040 (2009).

27. Y. Gan et al., "Automated classification of optical coherence tomography images of human atrial tissue," J. Biomed. Opt. 21(10), 101407 (2016).

28. M. Gargesha et al., "Parameter estimation of atherosclerotic tissue optical properties from three-dimensional intravascular optical coherence tomography," J. Med. Imaging 2(1), 016001 (2015).

29. T. Yonetsu et al., "Comparison of incidence and time course of neoatherosclerosis between bare metal stents and drug-eluting stents using optical coherence tomography," Am. J. Cardiol. 110(7), 933-939 (2012).

30. R. Nagoshi et al., "Qualitative and quantitative assessment of stent restenosis by optical coherence tomography: comparison between drug-eluting and bare-metal stents," Circ. J. 77(3), 652-660 (2013).

31. M. Gnanadesigan et al., "Optical coherence tomography attenuation imaging for lipid core detection: an ex-vivo validation study," Int. J. Cardiovasc. Imaging 33(1), 5-11 (2017).

32. G. J. Ughi et al., "Automated tissue characterization of in vivo atherosclerotic plaques by intravascular optical coherence tomography images," Biomed. Opt. Express 4(7), 1014-1030 (2013).
33. C. L. R. Rodriguez et al., "Decreased light attenuation in cerebral cortex during cerebral edema detected using optical coherence tomography," Neurophotonics 1(2), 025004 (2014).

34. G. T. Smith et al., "Automated, depth-resolved estimation of the attenuation coefficient from optical coherence tomography data," IEEE Trans. Med. Imaging 34(12), 2592-2602 (2015).

35. M. J. A. Girard et al., "Shadow removal and contrast enhancement in optical coherence tomography images of the human optic nerve head," Invest. Ophthalmol. Visual Sci. 52, 7738-7748 (2011).

36. J. M. Mari et al., "Enhancement of lamina cribrosa visibility in optical coherence tomography images using adaptive compensation," Invest. Ophthalmol. Visual Sci. 54, 2238-2247 (2013).

37. N. Foin et al., "Imaging of coronary artery plaques using contrastenhanced optical coherence tomography," Eur. Heart J.-Cardiovasc. Imaging 14(1), 85-85 (2013).

38. J. C. Teo et al., "Optimization of coronary optical coherence tomography imaging using the attenuation-compensated technique: a validation study," Eur. Heart J. Cardiovasc. Imaging 18(8), 880-887 (2016).

39. R. Lee et al., "Intravascular assessment of arterial disease using compensated OCT in comparison with histology," JACC: Cardiovasc. Imaging 9, 321-322 (2016).

40. B. D. Goldberg et al., "Performance of reduced bit-depth acquisition for optical frequency domain imaging," Opt. Express 17, 16957-16968 (2009).

41. S. Liu et al., "Light intensity matching between different intravascular optical coherence tomography systems," Proc. SPIE 9689, 96893D (2016).

42. J. E. Phipps et al., "Diagnosis of thin-capped fibroatheromas in intravascular optical coherence tomography images," Circ.: Cardiovasc. Interventions 9(7), e003163 (2016).

43. S. Liu et al., "Analysis and compensation for the effect of the catheter position on image intensities in intravascular optical coherence tomography," J. Biomed. Opt. 21(12), 126005 (2016).

44. A. Varkentin et al., "Simple model to simulate oct-depth signal in weakly and strongly scattering homogeneous media," J. Opt. 18(12), 125302 (2016).

45. J. J. R. Jimenez et al., "Automatic classification of atherosclerotic plaques imaged with intravascular OCT," Biomed. Opt. Express 7(10), 4069-4085 (2016).

46. A. Wang et al., "Automatic detection of bioresorbable vascular scaffold struts in intravascular optical coherence tomography pullback runs," Biomed. Opt. Express 5(10), 3589 (2014).

47. T. A. Rheude, E. Xhepa, and R. A. Byrne, "Case report markedly different tissue types on optical coherence tomography imaging in a patient with multiple lesion," Cathet. Cardiovasc. Interventions 89(6), E181E184 (2017).

48. H. Jia et al., "Effective anti-thrombotic therapy without stenting: intravascular optical coherence tomography-based management in plaque erosion (the erosionstudy)," Eur. Heart J. 38(11), 792-800 (2017).

Shengnan Liu received her BS degree in mathematics and applied mathematics in 2009 and her MS degree in controlling science and engineering in 2012. She is a PhD candidate at Leiden University Medical Center. Her current research interests include intensity analysis, optical properties, and tissue characterization with optical coherence tomography images.

Yohei Sotomi graduated from Osaka University and completed his cardiology training at Sakurabashi Watanabe Hospital, Japan. $\mathrm{He}$ is a clinical research fellow in cardiovascular medicine at the Academic Medical Center, University of Amsterdam, The Netherlands, and additionally works as a clinical researcher at Cardialysis, The Netherlands. His research interests include bioresorbable vascular scaffold, advanced intracoronary imaging (optical coherence tomography and intravascular ultrasound), and its clinical application.

Jeroen Eggermont received his MSc degree in computer science from Leiden University in 1998 and his PhD from Leiden University in 2005. He is a scientific researcher in the Division of Image Processing. Currently, his work is mainly focused on the automatic analysis of intravascular ultrasound and optical coherence tomography images. 
Gaku Nakazawa is an assistant professor of the Department of Cardiology at Tokai University. He has worked as an interventional cardiologist since 2003. During his career, he has worked in CVPath Institute as a research fellow and studied vascular pathology from 2006 to 2009, which resulted in publishing many manuscripts regarding pathology of coronary arteries and drug-eluting stents.

Sho Torii is a research associate at Tokai University. He has been working at the Division of Cardiology since 2011 and his main work is cardiovascular pathology.

Takeshi ljichi graduated from Tokai University and completed his cardiology training at the Tokai University School of Medicine. $\mathrm{He}$ is a clinical physician in cardiovascular medicine at Tokai University School of Medicine, Japan. His research interests include coronary imaging (optical coherence tomography and intravascular ultrasound), coronary pathology, cardiac regenerative therapy, and its clinical application.

Yoshinobu Onuma graduated from Tohoku University, Japan, and completed his cardiology fellowship at Mitsui Memorial Hospital. $\mathrm{He}$ is a supervising cardiologist at Cardialysis and works in the Throaxcenter at Erasmus Medical Center, The Netherlands. He was granted his doctorate in interventional cardiology at the Erasmus University in 2014. He is a leading expert in the field of bioresorbable scaffolds, metallic stents, quantitative coronary angiography, intravascular imaging, and multislice computer tomography.
Patrick W. Serruys is an emeritus professor of medicine at Erasmus Medical Center, Rotterdam, and a professor of cardiology at the Imperial College, London. He is also a Doctor Honoris Causa in medicine from the University of Athens; medicine at the Complutense University of Madrid; engineering of the Melbourne University. He has been working as a principal investigator of many clinical trials, resulting in an enormous number of publications and awards.

Boudewijn P. F. Lelieveldt is the head of the Division of Image Processing (LKEB), Department of Radiology, Leiden University Medical Center, Leiden, The Netherlands. $\mathrm{He}$ is also appointed as a medical delta professor at the Department of Intelligent Systems, Delft University of Technology, Delft, The Netherlands. He serves as a member of the editorial board of Medical Image Analysis and the International Journal of Cardiovascular Imaging, and is an associate editor of IEEE Transactions on Medical Imaging.

Jouke Dijkstra is an associate professor at LUMC-LKEB, where he is the leader of the vascular and molecular imaging section. He has been working at the Division of Image Processing since 1996 and is responsible for the research in vessel imaging, in particular intravascular ultrasound, optical coherence tomography, and computed tomographic angiography. The research resulted in several scientific publications and analytical software packages, which have been transferred to industry and are used world-wide. 